This item was submitted to Loughborough's Research Repository by the author.

Items in Figshare are protected by copyright, with all rights reserved, unless otherwise indicated.

\title{
Two properties of stochastic KPP equations: ergodicity and pathwise
} property

PLEASE CITE THE PUBLISHED VERSION

LICENCE

CC BY-NC-ND 4.0

\section{REPOSITORY RECORD}

Oksendal, B., G. Vage, and Huaizhong Zhao. 2019. "Two Properties of Stochastic KPP Equations: Ergodicity and Pathwise Property". figshare. https://hdl.handle.net/2134/762. 


\title{
Two Properties of Stochastic KPP Equations: Ergodicity and Pathwise Property
}

\author{
B. Øksendal, G. Våge and H. Z. Zhao
}

\begin{abstract}
In this paper we study the random approximate travelling wave solutions of the stochastic KPP equations. Two new properties of the stochastic KPP equations are obtained. We prove the ergodicity that for almost all sample paths, behind the wave front $x=\gamma t$, the lower limit of $\frac{1}{t} \int_{0}^{t} u(s, x) d s$ as $t \rightarrow \infty$ is positive, and ahead of the wave front, the limit is zero. In some cases, behind the wave front, the limit of $\frac{1}{t} \int_{0}^{t} u(s, x) d s$ as $t \rightarrow \infty$ exists and is positive almost surely. We also prove that behind the wave front, for almost each $\omega$, the solution of some special stochastic KPP equations converges to a stationary trajectory of the corresponding stochastic differential equation. In front of wave front, the solution converges to 0 which is another stationary trajectory of the corresponding SDE. We also study the space derivative of the solution for large time. We show that away from the wave front, for almost all large $t$ the solution is flat in the $x$-direction for almost all sample paths.
\end{abstract}

\section{Introduction}

Approximate random travelling wave solutions for stochastic KPP eqautions were studied by [7], [10] and [25]. These equations arise in the study of the effect of a noise to approximate travelling waves of semi-linear reaction diffusion equations. We say that the noise is mild if $k_{m}<\sqrt{2 c(0)}$, where $k_{m}=\lim _{t \rightarrow \infty} \sqrt{\frac{1}{t} \int_{0}^{t} k^{2}(s) d s}$, assuming the limit exists. It was known that in an environment of mild noise, the solution of the stochastic generalized KPP equation

$$
d u(t, x)=\left(\frac{D}{2} \Delta u(t, x)+c(u) u\right) d t+k(t) u d W_{t},
$$

still evolves to an approximate travelling wave solution with a reduced speed $\gamma$ $=\sqrt{D\left(2 c(0)-k_{m}^{2}\right)}$ in the limit as $t \rightarrow \infty$. It was proved under some conditions which will be specified in Section 3 that Equation (1.1) has a random travelling wave solution $([10],[7],[25])$. The wave front was known as

$$
x=\gamma t
$$


and there are constants $d_{1}, d_{2}, d_{3}>0$ such that for any $h>0$

$$
\frac{1}{t} \log u(t, x)<-d_{1} \text { if } x>(\gamma+h) t, \text { for almost all } \omega
$$

and

$$
-d_{3} \leq \frac{\log u(t, x)}{\sqrt{2 t \log \log t}} \leq d_{2} \text { if } x<(\gamma-h) t, \text { for almost all } \omega
$$

for all sufficiently large $t$.

The travelling wave of the FKPP equation (when $k=0$ and $c(u)=1-u$ ) was first studied by [18] and [12]. Since then, it has attracted many mathematicians' attentions (e.g. $[2],[3],[4],[5],[6],[9],[11],[13],[14],[16],[22],[24],[27]$, to name but a few.) It was defined as a solution of the type $u(t, x)=U(x-\gamma t)$ and $\gamma$ is the speed of the travelling wave. Here $U$ satisfies $\lim _{z \rightarrow-\infty} U(z)=1$ and $\lim _{z \rightarrow+\infty} U(z)=0$. Note that $\lim _{t \rightarrow \infty} u(t,(\gamma-h) t)=1$ and $\lim _{t \rightarrow \infty} u(t,(\gamma+h) t)=0$ for any $h>0$. This was extended to Freidlin's type approximate travelling wave for reaction diffusion equations by [13] so that it is applicable to much more general situations. And a variety of stochastic methods has been developed to study travelling waves $([5],[6],[9],[13],[14],[22])$. Approximate travelling waves in a random media were also studied in [13].

For the stochastic KPP equation (1.1), ahead of the wave front, the solution is exponentially small $\left(\leq \mathrm{e}^{-d_{1} t}\right)$ almost surely and behaves the same as Freidlin's approximated travelling wave. But behind the wave front, the solution is oscillatory. It was clear that the solution behind the wave front behaves differently from the solution ahead of the wave front as (1.3) and (1.4) have shown. We call the regions behind and ahead of the wave front the crest and the trough of the random approximate travelling wave, respectively. It was remained open to understand the behaviour of the solution on the crest beyond (1.4). It was pointed out in [10] suggested by the numerical works in [15] that in the limit $t \rightarrow \infty$, $\frac{1}{t} \int_{0}^{t} u(s, x) d s$ should have a simple formula. The integral $\int_{0}^{1} u^{\mu}(t, x) d t$ was simulated numerically in [15] for rescaled stochastic KPP equations. In this paper, we find a way to study the limit of $\frac{1}{t} \int_{0}^{t} u(s, x) d s$ as $t \rightarrow \infty$ for Equation (1.1). To explain this ergodicity result, we demonstrate here a special case of Equation (1.1) with $c(u)=c(1-u)$, the latter $c$ being a constant and $k(t)=k$ being a constant. In this case the equation is

$$
d u(t, x)=\left(\frac{D}{2} \Delta u(t, x)+c(1-u) u\right) d t+k u d W_{t},
$$

with initial condition $u(0, x)=\chi_{x \leq 0}$. Define $\gamma=\sqrt{D\left(2 c-k^{2}\right)}$. We have for any $h>0$,

$$
\lim _{t \rightarrow \infty} \frac{1}{t} \int_{0}^{t} \inf _{x \leq(\gamma-h) t} u(s, x) d s=\lim _{t \rightarrow \infty} \frac{1}{t} \int_{0}^{t} \sup _{x} u(s, x) d s=1-\frac{k^{2}}{2 c}, \quad \text { a.s.. }
$$

This result is new in the literature. It is easy to see from (1.3) that on the trough,

$$
\lim _{t \rightarrow \infty} \frac{1}{t} \int_{0}^{t} \sup _{x \geq(\gamma+h) t} u(s, x) d s=0, \quad \text { a.s.. }
$$


It is noted that these limits are true for almost all sample paths.

Our method is first to study the corresponding stochastic ordinary differential equation. In Section 2, we find its explicit solution $Y(t)$ to the SODE and thereafter we calculate $\lim _{t \rightarrow \infty} \frac{1}{t} \int_{0}^{t} Y(s) d s$. In Section 3, we use the Feynman-Kac formula to obtain a comparison result of the solution $u(t, x)$ to Equation (1.1) and $Y(t)$. So we can use the result in Section 2 to obtain $\lim _{t \rightarrow \infty} \frac{1}{t} \int_{0}^{t} u(s, x) d s$ on the crest.

On the other hand, we study the pathwise property of the solution of a special stochastic KPP equation (1.5). Note the corresponding stochastic differential equation

$$
d Y(t)=c(1-Y(t)) Y(t) d t+k Y(t) d W_{t}
$$

has two stationary trajectories in the sense of Mohammed and Scheutzow. These are 0 and $Z(\omega)$ given by

$$
Z(\omega)=\left(c \int_{-\infty}^{0} \exp \left\{\left(c-\frac{1}{2} k^{2}\right) s+k W_{s}\right\} d s\right)^{-1}
$$

We can check 0 is unstable and $Z(\omega)$ is stable by calculating the Lyapunov exponent at each point. The pathwise property for the stochastic KPP equation (1.5) we prove in this paper is that for any $h>0$, for $x<(\gamma-h) t$ and a.e. $\omega$,

$$
\left|u(t, x, \omega)-Z\left(\theta_{t}(\omega)\right)\right| \rightarrow 0 \text { as } t \rightarrow \infty
$$

and for $x>(\gamma+h) t$ and a.e. $\omega$,

$$
u(t, x, \omega) \rightarrow 0 \text { as } t \rightarrow \infty
$$

where $\theta_{t}$ is the canonical Brownian shift. Here (1.10) is new while the convergence to 0 result (1.11) was obtained in [7], [10] and [25]. We should point out here that we only obtain the pathwise result for stochastic KPP equation (1.5). But we expect this result is true for a wider class of the stochastic reaction diffusion equations.

In Section 4, we study the convergence of the space derivative of the solution. We prove that $\int_{0}^{t}|\nabla u(s, x)| d s$ converges as $t \rightarrow \infty$. Therefore for almost all large $t, \nabla u(t, x)$ is small for almost all $(t, x)$ on the crest and the trough. This result is established by considering the logarithmic derivative of the solution $R(t, x)=-\nabla \log u(t, x)$. The space derivatives of the logarithm of solutions for deterministic heat equations were studied by [20], [26]. The space derivatives for nonlinear KPP equations were studied by [19] and [28]. For the nonlinear generalized KPP equations, the fact that $u(t, x) \geq \delta$ on the crest for a constant $\delta>0$ played a crucial rule in the study of the space derivatives in [19] and [28]. But this is not true for the stochastic generalized KPP Equation (1.1). We find in this paper that $u(t, x) \geq \delta$ can be replaced by (1.6), which is a much weaker requirement. 


\section{Some preliminary results for certain related stochas- tic ordinary differential equations}

First we consider the following nonlinear stochastic ordinary differential equation,

$$
d Y(t, y)=\alpha Y(t, y)(\beta-Y(t, y)) d t+k(t) Y(t, y) d W_{t}, Y_{0}=y
$$

where $\alpha, \beta$, and $y>0$ are constants, $W_{t}$ is a Brownian motion defined on a probability space $(\Omega, \mathcal{F}, P), k(t)$ is a continuous function of $t$. It is easy to check Equation (2.1) has the explicit solution (see e.g. [1])

$$
Y(t, y)=\frac{\exp \left\{\alpha \beta t-\frac{1}{2} \int_{0}^{t} k^{2}(r) d r+\int_{0}^{t} k(r) d W_{r}\right\}}{\frac{1}{y}+\alpha \int_{0}^{t} \exp \left\{\alpha \beta s-\frac{1}{2} \int_{0}^{s} k^{2}(r) d r+\int_{0}^{s} k(r) d W_{r}\right\} d s}, \quad t \geq 0 .
$$

For simplicity, we assume that $\lim _{t \rightarrow \infty} \frac{1}{t} \int_{0}^{t} k^{2}(s) d s$ exists, and the limit is denoted by $k_{m}^{2}$.

Lemma 2.1 Let $Y(t)$ be the solution of Equation (2.1). If $\alpha \beta>\frac{1}{2} k_{m}^{2}$, then for any $\epsilon>0$, there exists $t_{0}>0$ such that,

$$
P\left\{\left|\frac{1}{t} \int_{0}^{t} Y(s) d s-\left(\beta-\frac{k_{m}^{2}}{2 \alpha}\right)\right|>\epsilon \text { for some } t>T\right\}<\exp \left\{-\frac{\alpha^{2} \epsilon^{2} T^{2}}{32 \int_{0}^{T} k^{2}(r) d r}\right\},
$$

for all $T>t_{0}$. In particular, as $t \rightarrow \infty$,

$$
\frac{1}{t} \int_{0}^{t} Y(s) d s \rightarrow \beta-\frac{k_{m}^{2}}{2 \alpha}
$$

almost surely.

Proof. It is easily seen that

$$
\int_{0}^{t} Y(s) d s=\frac{1}{\alpha} \log z(t)-\frac{1}{\alpha} \log z(0),
$$

where $z(t)=\frac{1}{y_{0}}+\alpha \int_{0}^{t} \exp \left(\alpha \beta s-\frac{1}{2} \int_{0}^{s} k^{2}(r) d r+\int_{0}^{s} k(r) d W_{r}\right) d s$. Define $z^{*}(t)=\int_{0}^{t} \exp (\alpha \beta s-$ $\left.\frac{1}{2} \int_{0}^{s} k^{2}(r) d r\right) d s$. Then it is easy to see that for any sufficiently small $\epsilon>0$, there exists $t_{0}^{*}>0$ such that for $t>t_{0}^{*}$,

$$
k_{m}^{2}-\frac{1}{8} \alpha \varepsilon \leq \frac{1}{t} \int_{0}^{t} k^{2}(s) d s \leq k_{m}^{2}+\frac{1}{8} \alpha \varepsilon,
$$

and

$$
\int_{0}^{t_{0}^{*}} \exp \left(\alpha \beta s-\frac{1}{2} \int_{0}^{s} k^{2}(r) d r\right) d s \leq M \exp \left(\alpha \beta t-\frac{1}{2} k_{m}^{2} t+\frac{1}{8} \alpha \varepsilon t\right)
$$


for a constant $M>0$ and

$$
\log \left(\frac{1}{\alpha \beta-\frac{1}{2} k_{m}^{2}+\frac{1}{8} \alpha \varepsilon}+M\right) \leq \frac{1}{8} \alpha \varepsilon t .
$$

And there exists $t_{0}^{* *}>t_{0}^{*}$ such that for $t>t_{0}^{* *}$,

$$
\exp \left(\alpha \beta t_{0}^{*}-\frac{1}{2} k_{m}^{2} t_{0}^{*}-\frac{1}{8} \alpha \varepsilon t_{0}^{*}\right) \leq \frac{1}{2} \exp \left(\alpha \beta t-\frac{1}{2} k_{m}^{2} t-\frac{1}{8} \alpha \varepsilon t\right),
$$

and

$$
\log \left(\frac{1}{2\left(\alpha \beta-\frac{1}{2} k_{m}^{2}-\frac{1}{8} \alpha \varepsilon\right)}\right) \geq-\frac{1}{8} \alpha \varepsilon t .
$$

Therefore for $t>t_{0}^{*}$, from (2.6) and (2.7),

$$
\begin{aligned}
z^{*}(t)= & \int_{0}^{t} \exp \left(\alpha \beta s-\frac{1}{2} \int_{0}^{s} k^{2}(r) d r\right) d s \\
= & \int_{0}^{t_{0}^{*}} \exp \left(\alpha \beta s-\frac{1}{2} \int_{0}^{s} k^{2}(r) d r\right) d s+\int_{t_{0}^{*}}^{t} \exp \left(\alpha \beta s-\frac{1}{2} \int_{0}^{s} k^{2}(r) d r\right) d s \\
\leq & \int_{0}^{t_{0}^{*}} \exp \left(\alpha \beta s-\frac{1}{2} \int_{0}^{s} k^{2}(r) d r\right) d s+\int_{t_{0}^{*}}^{t} \exp \left(\alpha \beta s-\frac{1}{2} k_{m}^{2} s+\frac{1}{8} \alpha \varepsilon s\right) d s \\
= & \int_{0}^{t_{0}^{*}} \exp \left(\alpha \beta s-\frac{1}{2} \int_{0}^{s} k^{2}(r) d r\right) d s \\
& +\frac{1}{\alpha \beta-\frac{1}{2} k_{m}^{2}+\frac{1}{8} \alpha \varepsilon}\left[\exp \left(\alpha \beta t-\frac{1}{2} k_{m}^{2} t+\frac{1}{8} \alpha \varepsilon t\right)-\exp \left(\alpha \beta t_{0}^{*}-\frac{1}{2} k_{m}^{2} t_{0}^{*}+\frac{1}{8} \alpha \varepsilon t_{0}^{*}\right)\right] \\
\leq & \left(\frac{1}{\alpha \beta-\frac{1}{2} k_{m}^{2}+\frac{1}{8} \alpha \varepsilon}+M\right) \exp \left(\alpha \beta t-\frac{1}{2} k_{m}^{2} t+\frac{1}{8} \alpha \varepsilon t\right) .
\end{aligned}
$$

And similarly, from (2.6) and (2.9), for $t>t_{0}^{* *}$,

$$
\begin{aligned}
z^{*}(t) & =\int_{0}^{t} \exp \left(\alpha \beta s-\frac{1}{2} \int_{0}^{s} k^{2}(r) d r\right) d s \\
& \geq \int_{t_{0}^{*}}^{t} \exp \left(\alpha \beta s-\frac{1}{2} \int_{0}^{s} k^{2}(r) d r\right) d s \\
& \geq \int_{t_{0}^{*}}^{t} \exp \left(\alpha \beta s-\frac{1}{2} k_{m}^{2} s-\frac{1}{8} \alpha \varepsilon s\right) d s \\
& =\frac{1}{\alpha \beta-\frac{1}{2} k_{m}^{2}-\frac{1}{8} \alpha \varepsilon}\left[\exp \left(\alpha \beta t-\frac{1}{2} k_{m}^{2} t-\frac{1}{8} \alpha \varepsilon t\right)-\exp \left(\alpha \beta t_{0}^{*}-\frac{1}{2} k_{m}^{2} t_{0}^{*}-\frac{1}{8} \alpha \varepsilon t_{0}^{*}\right)\right] \\
& \geq \frac{1}{2\left(\alpha \beta-\frac{1}{2} k_{m}^{2}-\frac{1}{8} \alpha \varepsilon\right)} \exp \left(\alpha \beta t-\frac{1}{2} k_{m}^{2} t-\frac{1}{8} \alpha \varepsilon t\right) .
\end{aligned}
$$

Then taking logarithm to (2.11) and (2.12), it is easy to see from (2.8) and (2.10) that for $t>t_{0}^{* *}$

$$
\left(\alpha \beta-\frac{k_{m}^{2}}{2}-\frac{1}{4} \alpha \epsilon\right) t \leq \log z^{*}(t) \leq\left(\alpha \beta-\frac{k_{m}^{2}}{2}+\frac{1}{4} \alpha \epsilon\right) t .
$$


Recall that $\frac{1}{\sqrt{\int_{0}^{t} k^{2}(r) d r}} \int_{0}^{s} k(r) d W_{r}(0 \leq s \leq t)$ is a time changed Brownian motion $\mathcal{X}\left(\frac{\int_{0}^{s} k^{2}(r) d r}{\int_{0}^{t} k^{2}(r) d r}\right)$. Here $\mathcal{X}(u)$ is a standard Brownian motion with of time $u$. Therefore $\mathcal{Y}(s)=$ $\int_{0}^{s} k(r) d W_{r}=\sqrt{\int_{0}^{t} k^{2}(r) d r} \mathcal{X}\left(\frac{\int_{0}^{s} k^{2}(r) d r}{\int_{0}^{t} k^{2}(r) d r}\right)$. Define $C_{1}=\log \alpha$ and $C_{2}=\log \left(\frac{1}{y_{0}}+\alpha\right)$. define

For any $\varepsilon>0$, take $t_{0} \geq t_{0}^{* *}$ such that $\frac{\left|C_{1}-\log y_{0}\right|}{\alpha t_{0}}<\frac{1}{2} \varepsilon$ and $\frac{\left|C_{2}-\log y_{0}\right|}{\alpha t_{0}}<\frac{1}{2} \varepsilon$. For any $T \geq t_{0}$,

$$
\Omega_{T}=\left\{\omega \in \Omega:-\frac{\alpha \varepsilon T}{4 \sqrt{\int_{0}^{T} k^{2}(r) d r}}<\mathcal{X}(u)<\frac{\alpha \varepsilon T}{4 \sqrt{\int_{0}^{T} k^{2}(r) d r}}, \text { for all } 0 \leq u \leq 1\right\},
$$

then from the wellknown Doob's inequality (see [21])

$$
P\left\{\Omega_{T}\right\}>1-\exp \left\{-\frac{\alpha^{2} \varepsilon^{2}}{32 \int_{0}^{T} k^{2}(r) d r} T^{2}\right\}
$$

and for each $\omega \in \Omega_{T}$, and $t \geq T$, and $s \leq t$,

$$
\begin{aligned}
|\mathcal{Y}(s)| & =\left|\int_{0}^{s} k(r) d W_{r}\right| \\
& =\sqrt{\int_{0}^{t} k^{2}(r) d r\left|\mathcal{X}\left(\frac{\int_{0}^{s} k^{2}(r) d r}{\int_{0}^{t} k^{2}(r) d r}\right)\right|} \\
& \leq \sqrt{\int_{0}^{t} k^{2}(r) d r} \frac{\alpha \varepsilon T}{4 \sqrt{\int_{0}^{T} k^{2}(r) d r}} \\
& \leq \frac{\alpha \varepsilon t}{4}
\end{aligned}
$$

for all $0 \leq s \leq t$. Then it follows easily that for $\omega \in \Omega_{T}$, and $t \geq T$,

$$
\alpha z^{*}(t) \mathrm{e}^{-\frac{1}{4} \alpha \varepsilon t} \leq z(t) \leq\left(\frac{1}{y_{0}}+\alpha\right) z^{*}(t) \mathrm{e}^{\frac{1}{4} \alpha \varepsilon t} .
$$

It turns out that for $\omega \in \Omega_{T}$, and $t \geq T$,

$$
\left(\alpha \beta-\frac{k_{m}^{2}}{2}-\frac{1}{2} \alpha \varepsilon\right) t+C_{1} \leq \log z(t) \leq\left(\alpha \beta-\frac{k_{m}^{2}}{2}+\frac{1}{2} \alpha \varepsilon\right) t+C_{2} .
$$

So from (2.5), for $\omega \in \Omega_{T}$, and $t \geq T$,

$$
\beta-\frac{k_{m}^{2}}{2 \alpha}-\frac{\epsilon}{2}+\frac{C_{1}-\log y_{0}}{\alpha t} \leq \frac{1}{t} \int_{0}^{t} Y(r) d s \leq \beta-\frac{k_{m}^{2}}{2 \alpha}+\frac{\varepsilon}{2}+\frac{C_{2}-\log y_{0}}{\alpha t} .
$$

Then by the definition of $t_{0}$, we have for $\omega \in \Omega_{T}$, and all $t \geq T\left(\geq t_{0}\right)$

$$
\beta-\frac{k_{m}^{2}}{2 \alpha}-\epsilon \leq \frac{1}{t} \int_{0}^{t} Y(r) d s \leq \beta-\frac{k_{m}^{2}}{2 \alpha}+\varepsilon .
$$


So (2.3) follows.

We need to generalize the above result to more general nonlinear SDEs. We have the following lemma. The key point of the proof is to use a comparison method.

Lemma 2.2 Suppose $c \in C\left(R^{+}\right)$, and there exist constants $a \geq b>0$ such that by $\leq$ $c(0)-c(y) \leq$ ay for all $y \geq 0$, and $c(0)>k_{m}^{2} / 2$. If $Y(t)$ denotes the solution of

$$
d Y(t)=Y(t) c(Y(t)) d t+k(t) Y(t) d W_{t}, Y_{0}=y_{0}
$$

then for any $\varepsilon>0$, there is $t_{0}=t_{0}(\varepsilon)>0$ such that,

$$
\begin{aligned}
& P\left\{\frac{1}{a}\left(c(0)-\frac{k_{m}^{2}}{2}\right)-\varepsilon \leq \frac{1}{t} \int_{0}^{t} Y(s) d s \leq \frac{1}{b}\left(c(0)-\frac{k_{m}^{2}}{2}\right)+\varepsilon \text { for all } t \geq T\right\} \\
>\quad & 1-\exp \left\{-\frac{a^{2} \varepsilon^{2} T^{2}}{32 \int_{0}^{T} k^{2}(r) d r}\right\}
\end{aligned}
$$

for all $T>t_{0}$. In particular, for a.e. $\omega$,

$$
\frac{1}{a}\left(c(0)-\frac{k_{m}^{2}}{2}\right) \leq \liminf _{t \rightarrow \infty} \frac{1}{t} \int_{0}^{t} Y(s) d s \leq \limsup _{t \rightarrow \infty} \frac{1}{t} \int_{0}^{t} Y(s) d s \leq \frac{1}{b}\left(c(0)-\frac{k_{m}^{2}}{2}\right) .
$$

Proof. If $c$ satisfies the conditions in the lemma, then

$$
a y\left(\frac{c(0)}{a}-y\right) \leq y c(y) \leq b y\left(\frac{c(0)}{b}-y\right)
$$

for all $y \geq 0$. The lemma now follows from the comparison theorem for SDE's (see e.g. [17]) and Lemma 2.1.

Remark 2.3 (i) We are only interested in the case $c(0)>\frac{1}{2} k_{m}^{2}$. It is easy to see that if $c(0)<\frac{1}{2} k_{m}^{2}$, then $Y(t) \rightarrow 0$ as $t \rightarrow \infty$ a.s.

(ii) If $\lim _{t \rightarrow \infty} \frac{1}{t} \int_{0}^{t} k^{2}(s) d s$ does not exist, we can denote $\underline{k}_{m}^{2}=\liminf _{t \rightarrow \infty} \frac{1}{t} \int_{0}^{t} k^{2}(s) d s$ and $\bar{k}_{m}^{2}=$ $\limsup _{t \rightarrow \infty} \frac{1}{t} \int_{0}^{t} k^{2}(s) d s$. If $\alpha \beta>\frac{1}{2} \bar{k}_{m}^{2}$, then the result is

$$
\beta-\frac{\bar{k}_{m}^{2}}{2 \alpha}=\liminf _{t \rightarrow \infty} \frac{1}{t} \int_{0}^{t} Y(s) d s \leq \limsup _{t \rightarrow \infty} \frac{1}{t} \int_{0}^{t} Y(s) d s=\beta-\frac{\underline{k}_{m}^{2}}{2 \alpha},
$$

almost surely. The results in next two sections are still valid with some necessary restatements as (2.18).

In next section, we will also need the following lemma. 
Lemma 2.4 Assume $c(y)$ is decreasing. Let $N(t)$ be a solution of

$$
\frac{d}{d t} N(t)=c(N(t)) N(t)-\frac{1}{2} k^{2}(t) N(t), \quad N(0)=y_{0} .
$$

Then there exists $t_{0}>0$ such that for $t>t_{0}$, the solution to (2.24) satisfies

$$
N(t) \mathrm{e}^{\inf _{0 \leq \Delta \leq t} \int_{\sigma}^{t} k(r) d W_{r}} \leq Y(t) \leq N(t) \mathrm{e}^{\sup ^{0 \leq \sigma \leq t} \int_{\sigma}^{t} k(r) d W_{r}} .
$$

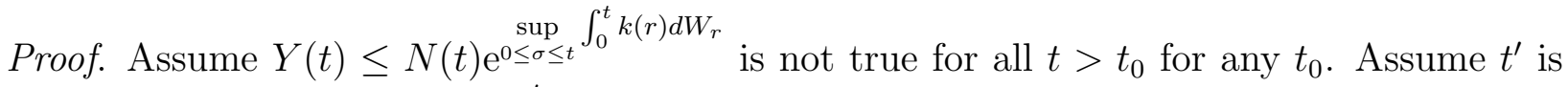

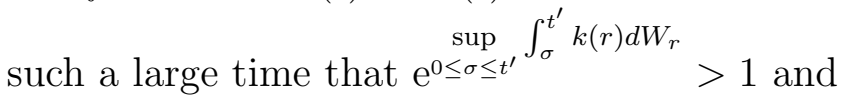

$$
Y\left(t^{\prime}\right)>N\left(t^{\prime}\right) \mathrm{e}^{\sup ^{0 \leq \sigma \leq t^{\prime}} \int_{\sigma}^{t^{\prime}} k(r) d W_{r}} .
$$

It is evident that $Y\left(t^{\prime}\right)>N\left(t^{\prime}\right)$. Define $\tau$ such that $Y(\tau)=N(\tau)$ and $Y(s) \geq N(s)$ for $\tau<s \leq t^{\prime}$. First note that

$$
N\left(t^{\prime}\right)=N(\tau) \mathrm{e}^{\int_{\tau}^{t^{\prime}} c(N(r)) d r-\frac{1}{2} \int_{\tau}^{t^{\prime}} k^{2}(r) d r} .
$$

Then it is easy to see that

$$
\begin{aligned}
Y\left(t^{\prime}\right) & =y_{0} \mathrm{e}^{\int^{t^{\prime}} c(Y(r)) d r-\frac{1}{2} \int_{0}^{t^{\prime}} k^{2}(r) d r+\int_{0}^{t^{\prime}} k(r) d W_{r}} \\
& =Y(\tau) \mathrm{e}^{\int_{\tau}^{t^{\prime}} c(Y(r)) d r-\frac{1}{2} \int_{\tau}^{t^{\prime}} k^{2}(r) d r+\int_{\tau}^{t^{\prime}} k(r) d W_{r}} \\
& \leq N(\tau) \mathrm{e}^{\int_{\tau}^{t^{\prime}} c(N(r)) d r-\frac{1}{2} \int_{\tau}^{t^{\prime}} k^{2}(r) d r} \mathrm{e}^{\mathrm{eup}^{0 \leq s \leq t^{\prime}} \int_{\sigma}^{t^{\prime}} k(r) d W_{r}} \\
& \leq N\left(t^{\prime}\right) \mathrm{e}^{\sup ^{0 \leq s \leq t^{\prime}} \int_{\sigma}^{t^{\prime}} k(r) d W_{r}} .
\end{aligned}
$$

This contradicts (2.21). Therefore the second inequality in (2.20) is proved. The first part in (2.20) can be proved similarly.

Consider equation (2.1) with constant $k$ in the remaining part of this section

$$
d Y(t, y)=\alpha Y(t, y)(\beta-Y(t, y)) d t+k Y(t, y) d W_{t}, Y_{0}=y
$$

Here $W$ is a one dimensional Brownian motion on Wiener space $(\Omega, \mathcal{F}, P)$. That is to say that $\Omega$ is the space of all continuous paths $\omega: R \rightarrow R$ given the topology of uniform convergence, $\mathcal{F}$ is the Borel $\sigma$-field, $P$ is the Wiener measure and $W$ is defined by $W(t, \omega)=$ $\omega(t)$. It is easy to see that the solution (2.2) of equation (2.24) defines a stochastic flow $Y(s):\left(R^{+} \cup\{0\}\right) \times \Omega \rightarrow R^{+} \cup\{0\}$. Recall the canonical Brownian shift

$$
\theta(t, \omega)(s)=\omega(t+s)-\omega(t) .
$$


Then by flow property we know that $W(t+s, \omega)=W\left(s, \theta_{t} \omega\right)+W(t, \omega)$. So

$$
W\left(s, \theta_{t} \omega\right)=W(t+s, \omega)-W(t, \omega)
$$

Define

$$
Z(\omega)=\left(\alpha \int_{-\infty}^{0} \exp \left\{\alpha \beta s-\frac{1}{2} k^{2} s+k W(s)\right\} d s\right)^{-1} .
$$

Simple calculation shows

$$
\begin{aligned}
Z\left(\theta_{t} \omega\right) & =\left(\alpha \int_{-\infty}^{0} \exp \left\{\alpha \beta s-\frac{1}{2} k^{2} s+k W(s+t, \omega)-k W(t, \omega)\right\} d s\right)^{-1} \\
& =\frac{\exp \left\{\alpha \beta t-\frac{1}{2} k^{2} t+k W(t, \omega)\right\}}{\alpha \int_{-\infty}^{0} \exp \left\{\alpha \beta(s+t)-\frac{1}{2} k^{2}(s+t)+k W(s+t, \omega)\right\} d s} \\
& =\frac{\exp \left\{\alpha \beta t-\frac{1}{2} k^{2} t+k W(t, \omega)\right\}}{\alpha \int_{-\infty}^{t} \exp \left\{\alpha \beta s-\frac{1}{2} k^{2} s+k W(s, \omega)\right\} d s}
\end{aligned}
$$

Then it is easy to check that

$$
Y(t, Z(\omega), \omega)=Z\left(\theta_{t}(\omega)\right) .
$$

According to Mohammed-Scheutzow's definition of stationary trajectory $([23]), Z(\omega)$ is a nontrivial stationary trajectory of equation (2.24). One can calculate the Lyapunov exponent of the linearized equation at the stationary trajectory (c.f. [1]). Moreover, for any $y>0$, consider the derivative flow $V_{t}=\mathrm{D} Y(t, y) V$ where $V$ being given and $V_{0}=V$. Here $\mathrm{D}$ is the derivative with respect to $y$. First note that $V_{t}$ satisfies the following linear stochastic differential equation, which can be obtained by differentiating equation (2.24),

$$
d V_{t}=\alpha(\beta-2 Y(t, y)) V_{t} d t+k V_{t} d W(t), V_{0}=V .
$$

Solving equation (2.28) we obtain

$$
V_{t}=V \exp \left\{\alpha \int_{0}^{t}(\beta-2 Y(s, y)) d s-\frac{1}{2} k^{2} t+k W(t)\right\} .
$$

It turns out that

$$
\begin{aligned}
\lim _{t \rightarrow \infty} \frac{1}{t} \log V_{t} & =\alpha \beta-\lim _{t \rightarrow \infty} \frac{2 \alpha}{t} \int_{0}^{t} Y(s, y) d s-\frac{1}{2} k^{2}+\lim _{t \rightarrow \infty} \frac{1}{t} k W(t) \\
& =-\left(\alpha \beta-\frac{k^{2}}{2}\right)<0, \quad \text { P.a.s.. }
\end{aligned}
$$

Here we used Lemma 2.1 to calculate $\lim _{t \rightarrow \infty} \frac{1}{t} \int_{0}^{t} Y(s, y) d s$. Similar to [23], there is a sure event $\Omega^{*} \in \mathcal{F}$ with $\theta(t,.) \Omega^{*}=\Omega^{*}$ for all $t \in R$ such that for each $\omega \in \Omega^{*}$, for any $y>0$, for the discretized flow $Y_{n}$,

$$
\begin{aligned}
\lim _{n \rightarrow \infty} \frac{1}{n} \log \left|Y(n, y, \omega)-Z\left(\theta_{n}(\omega)\right)\right| & =\lim _{n \rightarrow \infty} \frac{1}{n} \log |Y(n, y, \omega)-Y(n, Z(\omega), \omega)| \\
& =-\left(\alpha \beta-\frac{k_{m}^{2}}{2}\right)<0 .
\end{aligned}
$$


But according to [1] (P185), $E Z(\omega)<\infty$. Therefore by Jensen's inequality, $E \log ^{+} Z(\omega)<$ $E \log (1+Z(\omega)) \leq \log (1+E Z(\omega))<\infty$. That is to say the integrability condition of Lemma 3.4 in [23] is satisfied. Therefore we can apply the Lemma to pass (2.31) to continuous time to derive the following lemma.

Lemma 2.5 Let $Y(t, y)$ be the solution of stochastic differential equation (2.24), $Z(\omega)$ be defined by (2.26) and $\theta_{t}$ be the canonical Brownian shift. Then there is a sure event $\Omega^{*} \in \mathcal{F}$ with $\theta(t,.) \Omega^{*}=\Omega^{*}$ such that for any $y>0$, and for each $\omega \in \Omega^{*}$,

$$
\lim _{t \rightarrow \infty} \frac{1}{t} \log \left|Y(t, y, \omega)-Z\left(\theta_{t}(\omega)\right)\right|=-\left(\alpha \beta-\frac{k^{2}}{2}\right)<0 .
$$

\section{$3 \quad$ Ergodic property and pathwise property of the stochas- tic KPP equations}

We consider the following stochastic KPP equation

$$
d u(t, x)=\left(\frac{D}{2} \Delta u(t, x)+u(t, x) c(u(t, x))\right) d t+k(t) u(t, x) d W_{t},\left.u\right|_{t=0}=u_{0},
$$

where $u_{0}=\chi_{(-\infty, 0]}$. Here $W_{t}$ is a Brownian motion on the probability space $(\Omega, \mathcal{F}, P)$. Recall $k_{m}^{2}=\lim _{t \rightarrow \infty} \frac{1}{t} \int_{0}^{t} k^{2}(s) d s$ assuming the limit exists. Assume

Condition (i). Function $c(u)$ is continuous and $c(u) \leq c(0)$ and $c(u)<0$ for $u>1$.

Condition (ii). Function $c(u)$ is decreasing.

Condition (iii). There exist constants $a \geq b>0$ such that $b \xi \leq c(u)-c(u+\xi) \leq a \xi$ for any $\xi \geq 0$ and $u \geq 0$.

Condition (iv). $c(0)>\frac{1}{2} k_{m}^{2}$.

Let $B_{r}$ be a Brownian motion in $R^{1}$ on a probability space $(\hat{\Omega}, \hat{\mathcal{F}}, \hat{P})$. Note $B_{r}$ and $W_{r}$ are independent. Then $u(t, x)$ satisfies the following Feynman-Kac formula:

$$
\begin{aligned}
u(t, x)= & \hat{E} u\left(0, x+\sqrt{D} B_{t}\right) \\
& \quad \exp \left\{\int_{0}^{t} c\left(u\left(t-r, x+\sqrt{D} B_{r}\right)\right) d r-\frac{1}{2} \int_{0}^{t} k^{2}(r) d r+\int_{0}^{t} k(r) d W_{r}\right\} .
\end{aligned}
$$

Here $\hat{E}$ is the expectation with respect to the probability measure $\hat{P}$.

Recall that under Conditions (i), (ii) and (iv) ([10],[7],[25])

$$
\begin{aligned}
v(t, x) \exp \left(\inf _{0 \leq \sigma \leq t} \int_{\sigma}^{t} k_{s} d W_{s}\right) & \leq u(t, x, \omega) \\
& \leq v(t, x) \exp \left(\sup _{0 \leq \sigma \leq t} \int_{\sigma}^{t} k_{s} d W_{s}\right)
\end{aligned}
$$


where $v(t, x)$ is the solution of the following deterministic reaction diffusion equation

$$
\frac{\partial v}{\partial t}=\frac{D}{2} \Delta v+v\left(c(v)-\frac{1}{2} k^{2}(t)\right), v(0, \cdot, \omega)=\chi_{(-\infty, 0]} ;(t, x) \in R^{+} \times R .
$$

In this section, we prove some new results about the random travelling waves. First, from (1.3) it is trivial to see

$$
\lim _{t \rightarrow \infty} \frac{1}{t} \int_{0}^{t} u(s,(\gamma+h) s) d s=0
$$

for any $h>0$. We will investigate the same limit behind the wave front. But generally speaking, we don't know whether or not the limit actually exists except in some special cases (this will be clear later). However we will be able to give the upper limit and lower limit of $\frac{1}{t} \int_{0}^{t} u(s, x) d s$ on the crest as $t \rightarrow \infty$. The upper limit is straightforward.

Theorem 3.1 Suppose c satisfies Conditions (i), (ii) and (iii) and let $u$ be the solution of (3.1). Then for almost all $\omega$,

$$
\limsup _{t \rightarrow \infty} \frac{1}{t} \int_{0}^{t} \sup _{x \in R} u(s, x) d s \leq \frac{1}{b}\left(c(0)-\frac{k_{m}^{2}}{2}\right) .
$$

Proof. Let $Y(t)$ be the solution of $(2.24)$ with $Y(0)=1$. First note that $Y(t, x)=Y(t)$ is the solution of $(3.1)$ with the initial condition $Y(0, x)=1$. Therefore for any $\tau \leq t$, it is easy to see

$$
Y(t)=Y(t-\tau) \mathrm{e}^{\int_{0}^{\tau} c\left(Y_{t-r}\right) d r-\frac{1}{2} \int_{0}^{\tau} k^{2}(t-r) d r-\int_{0}^{\tau} k(t-r) d W_{t-r}} .
$$

We will prove $0 \leq u(t, x) \leq Y(t, x)=Y(t)$ for all $x \in R$. Assume it is wrong, then $u(t, x)>$ $Y(t)$ for certain $t$ and $x$. Define a stopping time $\tau=\inf \left\{r: u\left(t-r, x+\sqrt{D} B_{r}\right) \leq Y(t-r)\right\}$. Then by Markov property of Brownian motion, as $c$ is decreasing,

$$
\begin{aligned}
u(t, x)= & \hat{E} u\left(t-\tau, x+\sqrt{D} B_{\tau}\right) \\
& \exp \left\{\int_{0}^{\tau} c\left(u\left(t-r, x+\sqrt{D} B_{r}\right)\right) d r-\frac{1}{2} \int_{0}^{\tau} k^{2}(t-r) d r-\int_{0}^{\tau} k(t-r) d W_{t-r}\right\} \\
\leq & Y(t-\tau) \exp \left\{\int_{0}^{\tau} c(Y(t-r)) d r-\frac{1}{2} \int_{0}^{\tau} k^{2}(t-r) d r-\int_{0}^{\tau} k(t-r) d W_{t-r}\right\} \\
= & Y(t) .
\end{aligned}
$$

Therefore we have desored inequality. The result now follows from Lemma 2.2.

The proof of a lower bound is more complicated. The following lemma is needed.

Lemma 3.2 Assume Conditions (i) and (ii), then a.s.

$$
\lim _{t \rightarrow \infty} \frac{1}{t} \log u\left(t, t \sqrt{\left(2 c(0)-k_{m}^{2}\right) D}\right)=0 .
$$


Proof. Let $v$ be the solution of (3.4). Recall (e.g. [9]) that for any $\epsilon>0$, there exists $t_{0}>0$ such that

$$
-\frac{\varepsilon t}{2} \leq \log v\left(t, t \sqrt{\left(2 c(0)-k_{m}^{2}\right) D}\right) \leq \frac{\varepsilon t}{2}, t \geq t_{0} .
$$

Similar to the proof of Lemma 2.1, for any $T \geq t_{0}$, there exists $\Omega_{T} \subset \Omega$ with $P\left\{\Omega_{T}\right\}>$ $1-\exp \left\{-\frac{\varepsilon^{2}}{8 \int_{0}^{T} k^{2}(r) d r} T^{2}\right\}$, such that for each $\omega \in \Omega_{T}$, and $t \geq T$,

$$
\left|\int_{0}^{s} k(r) d W_{r}\right| \leq \frac{\varepsilon t}{2}
$$

for all $0 \leq s \leq t$. It follows from (3.3) that for all $t \geq T$ and $\omega \in \Omega_{T}$,

$$
-\varepsilon<\frac{1}{t} \log u\left(t, t \sqrt{\left(2 c(0)-k_{m}^{2}\right) D}\right)<\varepsilon,
$$

which completes the proof.

Lemma 3.3 Suppose c satisfies Conditions (i), (ii), (iii) and (iv) and let $u(t, x)$ be the solution of (3.1) and $Y(t)$ be the solution of (2.16) with $Y(0)=1$. Let $h>0$ be arbitrary. Then for any $\lambda$, there exist $t_{0}>0$ and $\delta>0$ such that

$$
P\left\{\inf _{x<t(\gamma b / a-h)} \frac{u(t, x)}{Y(t)} \geq 1-\lambda, \text { for all } t \geq T\right\}>1-\exp \{-\delta T\},
$$

for any $T \geq t_{0}$.

Proof. Let $h \in\left(0, \gamma \frac{b}{2 a}\right)$. For any $\lambda>0$, let $\epsilon>0$ be small enough such that

$$
\lambda h\left(c(0)-\frac{k_{m}^{2}}{2}\right) /(4 a)-b \lambda \epsilon-2 \epsilon \geq 0 .
$$

From Lemma 2.2, we know there exists $t_{0}^{*}>0$ such that for any $T \geq t_{0}^{*}$, there exists $\Omega_{T}^{1, \epsilon} \subset \Omega$ with $P\left(\Omega_{T}^{1, \varepsilon}\right)>1-\mathrm{e}^{-\frac{a^{2} \varepsilon^{2}\left(\frac{b}{2 a}\right)^{2} T^{2}}{32 \int_{0}^{\frac{b}{2 a} T} k^{2}(r) d r}}$ such that for any $\omega \in \Omega_{T}^{1, \varepsilon}$, and for all $s \geq \frac{b}{2 a} T$

$$
\frac{1}{a}\left(c(0)-\frac{k_{m}^{2}}{2}\right)-\varepsilon \leq \frac{1}{s} \int_{0}^{s} Y(r) d r \leq \frac{1}{b}\left(c(0)-\frac{k_{m}^{2}}{2}\right)+\varepsilon
$$

From Lemma 3.2 and Lemma 2.4, we know there exist $t_{1}=t_{1}(\epsilon(\lambda))$ and $\Omega_{T}^{2, \epsilon} \subset \Omega$ for all $T \geq t_{1}$, with $P\left(\Omega_{T}^{2, \epsilon}\right)>1-\mathrm{e}^{-\delta_{2} T}$ for a constant $\delta_{2}>0$, such that if $\omega \in \Omega_{T}^{2, \epsilon}$,

$$
u(s, \gamma s) \geq \mathrm{e}^{-\epsilon s}, \text { for all } s \geq T,
$$

and

$$
\mathrm{e}^{-\epsilon s} \leq Y(s)(\omega) \leq \mathrm{e}^{\epsilon s}, \text { for all } s \geq T
$$


It is trivial to see that there is a $t_{2}>0$ such that for all $s \geq t_{2}$,

$$
\mathrm{e}^{-\frac{h^{2} s}{8 D\left(1-\frac{b}{a}+\frac{h}{2 \gamma}\right)}}+\mathrm{e}^{-\frac{\left(h-\frac{b}{2 a} \gamma\right)^{2}}{2 D} s} \leq \frac{\lambda}{4}
$$

Define $t_{0}=\max \left\{\frac{2 a}{b} t_{0}^{*}, t_{1}, t_{2}\right\}$. Let $T \geq t_{0}$ be arbitrary. Define $\Omega_{T}^{0, \epsilon}=\Omega_{T}^{1, \epsilon} \cap \Omega_{T}^{2, \epsilon}$. First, it is evident that there is a $\delta>0$ such that $P\left(\Omega_{T}^{0, \epsilon}\right)>1-\mathrm{e}^{-\delta T}$ and for any $\omega \in \Omega_{T}^{0, \epsilon}$ and $s \geq T$, we have (3.12), (3.13-3.15). Now we shall show that for all $\omega \in \Omega_{T}^{0, \varepsilon}$, and all $t \geq T$,

$$
\inf _{x<t(\gamma b / a-h)} \frac{u(t, x)}{Y(t)} \geq 1-\lambda .
$$

Suppose (3.16) is false, i.e., there exist $\lambda>0, \omega \in \Omega_{T}^{0, \epsilon}$ and $t \geq T, x^{*}=x^{*}(t)<t(\gamma b / a-h)$ such that $u\left(t, x^{*}(t), \omega\right) \leq(1-\lambda) Y(t, \omega)$. Here $T$ and $\Omega_{T}^{0, \epsilon}$ are defined as above. Since $u(t, \cdot, \omega)$ is decreasing we have that

$$
0 \leq u\left(t,\left(\frac{\gamma b}{a}-h\right) t, \omega\right) \leq u\left(t, x^{*}(t), \omega\right) \leq(1-\lambda) Y(t, \omega) .
$$

For such a $t$ and $\omega$, define a stopping time

$$
\hat{\tau}^{t}=\inf \left\{r \geq 0 ; \hat{X}_{r}^{t} \notin F\right\}
$$

where

$$
\hat{X}_{r}^{t}=\left(t-r,(\gamma b / a-h) t+\sqrt{D} B_{r}\right)
$$

Here

$$
F=F_{t}=\left\{(s, x) ; s>0, x<\gamma s, u(s, x) \leq\left(1-\frac{\lambda}{2}\right) Y(s)\right\} .
$$

Note $\tau<t$ as $u(0, x)=1=Y_{0}$ for $x<0$ therefore line $s=0$ lies out side of $F$.

The boundary of $F$ consists of two parts. We denote the part along $x=\gamma s$ by $\partial F_{1}$ and the remaining part by $\partial F_{2}$. Define

$$
\begin{aligned}
& \hat{\Omega}_{1}=\left\{\hat{\omega} \in \hat{\Omega} ; \hat{X}_{\hat{t}^{t}}^{t} \in \partial F_{1}, \hat{\tau}^{t} \notin[(1-b / a+h / 2 a) t,(1-b / 2 a) t]\right\} \\
& \hat{\Omega}_{2}=\left\{\hat{\omega} \in \hat{\Omega} ; X_{\hat{\tau}^{t}}^{t} \in \partial F_{1}, \hat{\tau}^{t} \in[(1-b / a+h / 2 a) t,(1-b / 2 a) t]\right\}, \\
& \hat{\Omega}_{3}=\left\{\hat{\omega} \in \hat{\Omega} ; \hat{X}_{\hat{\tau}^{t}}^{t} \in \partial F_{2}\right\} .
\end{aligned}
$$

From the Feynman-Kac formula for $u(t, x)$ and the strong Markov property of Brownian motions, we see that

$$
\begin{aligned}
& u(t,(\gamma b / a-h) t, \omega) \\
= & \hat{E}\left[u\left(\hat{X}_{\hat{\tau}^{t}}^{t}\right) \exp \left(\int_{0}^{\hat{\tau}^{t}} c\left(u\left(\hat{X}_{r}^{t}, \omega\right)\right) d r+\int_{t-\hat{\tau}^{t}}^{t} k(r) d W_{r}(\omega)-\frac{1}{2} \int_{t-\hat{\tau}^{t}}^{t} k^{2}(r) d r\right)\right] \\
= & \sum_{i=1}^{3} u_{i}(t,(\gamma b / a-h) t, \omega),
\end{aligned}
$$


where

$$
\begin{aligned}
& u_{i}(t,(\gamma b / a-h) t, \omega) \\
= & \hat{E} \chi_{\hat{\Omega}_{i}} u\left(\hat{X}_{\hat{\tau}^{t}}^{t}, \omega\right) \\
& \times \exp \left(\int_{0}^{\hat{\tau}^{t}} c\left(u\left(\hat{X}_{r}^{t}, \omega\right)\right) d r+\int_{t-\hat{\tau}^{t}}^{t} k(r) d W_{r}(\omega)-\frac{1}{2} \int_{t-\hat{\tau}^{t}}^{t} k^{2}(r) d r\right),
\end{aligned}
$$

for $i=1,2,3$. We shall obtain lower bound on each of the $u_{i}$ 's . It is trivial to see that

$$
u_{1}(t, x) \geq 0, \text { for any }(t, x) .
$$

To obtain a lower bound on $u_{2}(t,(\gamma b / a-h) t)$ we start by considering the first integral in the exponential. Since $u(r, x) \leq(1-\lambda / 2) Y(r)$ for $(r, x) \in F$, from Conditions (ii) and (iii), it is easy to see

$$
\int_{0}^{\hat{\tau}^{t}} c\left(u\left(\hat{X}_{r}^{t}\right)\right) d r \geq \int_{0}^{\hat{\tau}^{t}} c\left((1-\lambda / 2) Y_{t-r}\right) d r \geq \int_{0}^{\hat{\tau}^{t}} c\left(Y_{t-r}\right) d r+\frac{b \lambda}{2} \int_{0}^{\hat{\tau}^{t}} Y_{t-r} d r .
$$

Note if $s \in\left[\left(1-\frac{b}{a}+\frac{h}{2 a}\right) t,\left(1-\frac{b}{2 a}\right) t\right]$, then $\frac{b}{2 a} t \leq t-s \leq\left(\frac{b}{a}-\frac{h}{2 a}\right) t$. It is evident that as $\omega \in \Omega_{T}^{1, \epsilon}, t \geq T$

$$
\begin{aligned}
\frac{b \lambda}{2} \int_{0}^{s} Y(t-r) d r & =\frac{b \lambda}{2}\left(\int_{0}^{t} Y(r) d r-\int_{0}^{t-s} Y(r) d r\right) \\
& \geq \frac{b \lambda}{2}\left[\left(\frac{1}{a}\left(c(0)-\frac{k_{m}^{2}}{2}\right)-\epsilon\right) t-\left(\left(\frac{1}{b}\left(c(0)-\frac{k_{m}^{2}}{2}\right)+\epsilon\right)\left(\frac{b}{a}-\frac{h}{2 a}\right) t\right]\right. \\
& =\frac{b \lambda}{2}\left[\frac{h}{2 a b}\left(c(0)-\frac{k_{m}^{2}}{2}\right) t-\epsilon t-\epsilon\left(\frac{b}{a}-\frac{h}{2 a}\right) t\right] \\
& \geq \frac{\lambda h}{4 a}\left(c(0)-\frac{k_{m}^{2}}{2}\right) t-b \lambda \epsilon t
\end{aligned}
$$

Since $Y(t)$ satisfies $(3.1)$ with $u_{0} \equiv 1$, there is an implicit Feynman-Kac formula for $Y(t)$. Using the strong Markov property as above we find that

$$
\exp \left(\int_{0}^{\hat{\tau}^{t}} c\left(Y_{t-r}\right) d r+\int_{t-\hat{\tau}^{t}}^{t} k(r) d W_{r}-\frac{1}{2} \int_{t-\hat{\tau}^{t}}^{t} k^{2}(r) d r\right)=Y(t)\left(Y\left(t-\hat{\tau}^{t}\right)\right)^{-1} .
$$

Recall (3.13) and (3.14). As $t \geq 2 a T / b$, so

$$
u\left(t-\hat{\tau}^{t}, \gamma\left(t-\hat{\tau}^{t}\right)\right) \geq e^{-\epsilon\left(t-\hat{\tau}^{t}\right)}>e^{-\epsilon t} \text { for all } \hat{\omega} \in \hat{\Omega}_{2},
$$

and

$$
e^{-\epsilon\left(t-\hat{\tau}^{t}\right)} \leq Y\left(t-\hat{\tau}^{t}\right)(\omega) \leq e^{\epsilon\left(t-\hat{\tau}^{t}\right)} .
$$


Hence for $\omega \in \Omega_{T}^{0, \varepsilon}$, and $t \geq T$, from (3.18),(3.20-3.22),

$$
\begin{aligned}
u_{2}(t,(\gamma b / a-h) t) & \geq \hat{E} \chi_{\hat{\Omega}_{2}} Y(t)\left(Y\left(t-\hat{\tau}^{t}\right)\right)^{-1} e^{\lambda h t\left(c(0)-k_{m}^{2} / 2\right) /(4 a)-b \lambda \epsilon t-\epsilon t} \\
& \geq \hat{P}\left(\hat{\Omega}_{2}\right) Y(t) e^{\left(\lambda h\left(c(0)-k_{m}^{2} / 2\right) /(4 a)-b \lambda \epsilon-2 \epsilon\right) t} \\
& \geq \hat{P}\left(\hat{\Omega}_{2}\right) Y(t)
\end{aligned}
$$

We also have that

$$
\begin{aligned}
u_{3}(t,(\gamma b / a-h) t) & \geq \hat{E} \chi_{\hat{\Omega}_{3}}\left(1-\frac{\lambda}{2}\right) Y\left(t-\hat{\tau}^{t}\right) \mathrm{e}^{\int_{0}^{\hat{\tau}^{t}} c\left(Y_{t-r}\right) d r+\int_{t-\hat{\tau}^{t}}^{t} k(r) d W_{r}-\int_{t-\hat{\tau}^{t}}^{t} k(r)^{2} d r / 2} \\
& \quad \mathrm{e}^{b \lambda \int_{0}^{\hat{\tau}^{t}} Y_{t-r} d r / 2} \\
& \geq \hat{P}\left(\hat{\Omega}_{3}\right)\left(1-\frac{\lambda}{2}\right) Y_{t}
\end{aligned}
$$

since $Y(t, \omega)$ satisfies an implicit Feynman-Kac formula (3.22) and $\int_{0}^{s} Y_{t-r} d r \geq 0$ for all $s \in[0, t]$.

Note if $\hat{\omega} \in \hat{\Omega}_{1}$ and $\tau<\left(1-\frac{b}{a}+\frac{h}{2 \gamma}\right) t$, then $X_{s}$ has to meet $x=\gamma s$ at a time $\tau \leq\left(1-\frac{b}{a}+\frac{h}{2 \gamma}\right) t$. Therefore $\left(\gamma \frac{b}{a}-h\right) t+\sqrt{D} B_{\tau} \geq \gamma\left(\frac{b}{a}-\frac{h}{2 \gamma}\right) t$. This is followed by $\sqrt{D} B_{\tau}>\frac{h}{2} t$. It turns out from Doob's inequality (see [21]) that

$$
\begin{aligned}
& \hat{P}\left\{\hat{\omega} \in \hat{\Omega}_{1} \text { and } \tau<\left(1-\frac{b}{a}+\frac{h}{2 \gamma}\right) t\right\} \\
\leq & \hat{P}\left\{\sqrt{D} B_{\tau}>\frac{h}{2} t, \text { and } \tau<\left(1-\frac{b}{a}+\frac{h}{2 \gamma}\right) t\right\} \\
\leq & \hat{P}\left\{\sup _{0 \leq s \leq\left(1-\frac{b}{a}+\frac{h}{2 \gamma}\right) t} \sqrt{D} B_{s}>\frac{h}{2} t\right\} \leq \exp \left\{-\frac{\left(\frac{h}{2} t\right)^{2}}{2 D\left(1-\frac{b}{a}+\frac{h}{2 \gamma}\right) t}\right\} \\
= & \exp \left\{-\frac{h^{2} t}{8 D\left(1-\frac{b}{a}+\frac{h}{2 \gamma}\right)}\right\} .
\end{aligned}
$$

Similarly, if $\hat{\omega} \in \hat{\Omega}_{1}$ and $\tau>\left(1-\frac{b}{2 a}\right) t$, then $X_{s}$ has to meet $x=\gamma s$ at a time $\tau>\left(1-\frac{b}{2 a}\right) t$. Therefore $\left(\gamma \frac{b}{a}-h\right) t+\sqrt{D} B_{\tau} \leq \gamma\left(\frac{b}{2 a}\right) t$. This is followed by $\sqrt{D} B_{\tau} \leq\left(h-\frac{b}{2 a}\right) t$. It turns out that

$$
\begin{aligned}
& \hat{P}\left\{\hat{\omega} \in \hat{\Omega}_{1} \text { and } \tau>\left(1-\frac{b}{2 a}\right) t\right\} \\
\leq & \hat{P}\left\{\sqrt{D} B_{\tau} \leq\left(h-\frac{b}{2 a}\right) t \text { and }\left(1-\frac{b}{2 a}\right) t \leq \tau \leq t\right\} \\
\leq & \hat{P}\left\{\inf _{0 \leq s \leq t} \sqrt{D} B_{s}<\left(h-\frac{b}{2 a} \gamma\right) t\right\} \\
\leq & \exp \left\{-\frac{\left(h-\frac{b}{2 a} \gamma\right)^{2}}{2 D} t\right\} .
\end{aligned}
$$


Recall (3.15). It is obvious that (3.27) and (3.28) imply $\hat{P}\left(\hat{\Omega}_{1}\right) \leq \frac{\lambda}{4}$ as $t \geq T$. It follows from (3.18), (3.19), (3.25) and (3.26) that for $\omega \in \Omega_{T}^{0, \varepsilon}$, and $t \geq T$

$$
\begin{aligned}
u(t,(\gamma b / a-h) t) & =\sum_{i=1}^{3} u_{i}(t,(\gamma b / a-h) t) \\
& \geq\left(1-\frac{\lambda}{2}\right) Y(t)\left(\hat{P}\left(\hat{\Omega}_{2}\right)+\hat{P}\left(\hat{\Omega}_{3}\right)\right) \\
& =\left(1-\frac{\lambda}{2}\right) Y(t)-\left(1-\frac{\lambda}{2}\right) Y(t) \hat{P}\left(\hat{\Omega}_{1}\right) \\
& =\left(1-\frac{\lambda}{2}\right) Y(t)-\frac{\lambda}{4} Y(t) \\
& \geq\left(1-\frac{3 \lambda}{4}\right) Y(t),
\end{aligned}
$$

if $T$ is sufficiently large. This contradicts (3.17). Therefore we have proved that for any $\lambda>0$, and sufficiently large $T$, if $\omega \in \Omega_{T}^{0, \varepsilon(\lambda)}$ and and $t \geq T$,

$$
\inf _{x<t(\gamma b / a-h)} u(t, x) \geq(1-\lambda) Y(t) .
$$

Theorem 3.4 Suppose c satisfies Conditions (i), (ii), (iii) and (iv) and u solves the SPDE (3.1). Let $h>0$ be arbitrary. For any $\varepsilon>0$, there exist $t_{0}=t_{0}(\varepsilon)$ and $\delta(\varepsilon)>0$ such that,

$$
P\left\{\frac{1}{t} \int_{0}^{t} \inf _{x<s(\gamma b / a-h)} u(s, x) d s>\frac{1}{a}\left(c(0)-\frac{k_{m}^{2}}{2}\right)-\varepsilon \text { for all } t \geq T\right\}>1-\exp \{-\delta T\},
$$

for all $T \geq t_{0}$

Proof. According to Lemma 3.3 and Lemma 2.2, there exist $t_{1}, \delta_{1}>0$ and $\Omega_{1, T}^{\varepsilon} \subset \Omega$ with $P\left(\Omega_{1, T}^{\varepsilon}\right)>1-\exp \left\{-\delta_{1} T\right\}$ for any $T \geq t_{1}$ such that for all $s \geq T$ and $\omega \in \Omega_{1, T}^{\varepsilon}$,

$$
\inf _{x<s(\gamma b / a-h)} u(s, x)>\left(1-\frac{a \varepsilon}{3\left(c(0)-\frac{k_{m}^{2}}{2}\right)}\right) Y(s),
$$

and

$$
\frac{1}{t} \int_{0}^{t} Y(s) d s \geq \frac{1}{a}\left(c(0)-\frac{k_{m}^{2}}{2}\right)-\frac{\varepsilon}{3} .
$$

Let $t_{0}>t_{1}$ satisfy

$$
\frac{1}{t_{0}} \ln 2+\frac{c(0) t_{1}+t_{0}^{\frac{1}{2}}}{t_{0}}<\frac{\varepsilon}{3}
$$


Then for any $T \geq t_{0}$, define

$$
\Omega_{2, T}=\left\{\omega \in \Omega: \sup _{0 \leq s \leq t_{1}} \int_{0}^{s} k(r) d W(r) \leq T^{\frac{1}{2}}\right\}
$$

It is easy to see that $P\left(\Omega_{2, T}\right)>1-\exp \left\{-\delta_{2} T\right\}$ for a constant $\delta_{2}>0$. Moreover, for all $\omega \in \Omega_{2, T}$ with $T \geq t_{0}$,

$$
\frac{1}{t} \int_{0}^{t_{1}} Y(s) d s<\frac{\varepsilon}{3}
$$

for all $t \geq T$.

So for $T \geq t_{0}$, define $\Omega_{T}=\Omega_{1, T} \cap \Omega_{2, T}$. It is easy to see $P\left(\Omega_{T}\right)>1-\exp \{-\delta T\}$ for a costant $\delta>0$ and $\omega \in \Omega_{T}$,

$$
\begin{aligned}
\frac{1}{t} \int_{0}^{t} \inf _{x<s(\gamma b / a-h)} u(s, x) d s & \geq \frac{1}{t} \int_{t_{1}}^{t} \inf _{x<s(\gamma b / a-h)} u(s, x) d s \\
& \geq\left(1-\frac{a \varepsilon}{3\left(c(0)-\frac{k_{m}^{2}}{2}\right)}\right) \frac{1}{t} \int_{t_{1}}^{t} Y(s) d s \\
& =\left(1-\frac{a \varepsilon}{3\left(c(0)-\frac{k_{m}^{2}}{2}\right)}\right) \frac{1}{t}\left(\int_{0}^{t} Y(s) d s-\int_{0}^{t_{1}} Y(s) d s\right) \\
& \geq\left(1-\frac{a \varepsilon}{3\left(c(0)-\frac{k_{m}^{2}}{2}\right)}\right)\left\{\frac{1}{a}\left(c(0)-\frac{k_{m}^{2}}{2}\right)-\frac{\varepsilon}{3}-\frac{\varepsilon}{3}\right\} \\
& >\frac{1}{a}\left(c(0)-\frac{k_{m}^{2}}{2}\right)-\varepsilon,
\end{aligned}
$$

for all $t \geq T$. Here we have used (3.32), (3.33) and (3.36). The proof is completed.

Remark 3.5 (i). Although the solutions of the stochastic generalized KPP equations may not converge as time and space tend to $\infty$, Theorem 3.4 tells us that the time average grows linearly with time for large time. In some special case, $\frac{1}{t} \int_{0}^{t} u(s, x) d s$ converges on the crest. For this see Remark 3.5 (ii). It is trivial to see $\frac{1}{t} \int_{0}^{t} u(s, x) d s$ converges to zero on the trough.

(ii). Note that if $a=b$, i.e. if $c$ is linear, we find that for any $h>0$ almost all $\omega \in \Omega$,

$$
\lim _{t \rightarrow \infty} \frac{1}{t} \int_{0}^{t} \inf _{x<(\gamma-h) s} u(s, x) d s=\lim _{t \rightarrow \infty} \frac{1}{t} \int_{0}^{t} \sup _{x \in R} u(s, x) d s=c(0)-\frac{k_{m}^{2}}{2} .
$$

Now we consider the following stochastic KPP equation (3.1) when $c(u)=c(1-u)$, and $k$ is a constant

$$
d u(t, x)=\left(\frac{D}{2} \Delta u(t, x)+c u(t, x)(1-u(t, x))\right) d t+k u(t, x) d W_{t},\left.u\right|_{t=0}=u_{0},
$$

where $u_{0}=\chi_{(-\infty, 0]}$. Here $W_{t}$ is a Brownian motion on the Wiener space $(\Omega, \mathcal{F}, P)$, and $c$ is a constant. 
Theorem 3.6 Let $u(t, x)$ be the solution of (3.39) and $\theta_{t}$ be the canonical Brownian shift. Then

$$
\lim _{t \rightarrow \infty} \sup _{x<(\gamma-h) t}\left|u(t, x, \omega)-Z\left(\theta_{t} \omega\right)\right|=0, \quad \text { P.a.s }
$$

for any $h>0$. Here $\gamma=\sqrt{2 D\left(c-\frac{k_{m}^{2}}{2}\right)}$ and $Z(\omega)$ is given by

$$
Z(\omega)=\left(c \int_{-\infty}^{0} \exp \left\{\left(c-\frac{1}{2} k^{2}\right) s+k W(s)\right\} d s\right)^{-1} .
$$

Proof. Let $Y(t)=Y(t, 1)$. The proof follows from Lemma 2.5, Lemma 3.3 and the inquality $u(t, x) \leq Y(t)$ immediately.

\section{Behaviour of the derivatives}

It is easy to see that the solution $u(t, x)$ is $C^{2}$ in $x$ for all $t>0$ and almost all $\omega \in \Omega$ (see [25]). Let $R(t, x)=-D \nabla \log u(t, x)$ for $t>0$, then it is easy to verify that $R(t, x)$ satisfies the following Burgers' equation for $t>0$ and a.e. $\omega \in \Omega$ :

$$
\frac{\partial}{\partial t} R(t, x)+R(t, x) \nabla R(t, x)=\frac{D}{2} \Delta R(t, x)+c^{\prime}(u(t, x)) u(t, x) R(t, x) .
$$

Let $y_{r}$ be the solution of the following stochastic differential equation up to explosion time

$$
d y_{r}=\sqrt{D} d B_{r}-R\left(t-r, y_{r}\right) d r, \quad y_{0}=x .
$$

Here $B_{r}$ is a Brownian motion on the probability space $\left(\hat{\Omega}, \hat{\mathcal{F}}, \hat{\mathcal{F}}_{r}, \hat{P}\right\}$. See [8] for explosion time.

We first prove that $y_{r}$ is nonexplosive.

Lemma 4.1 Assume Conditions (i) and (ii). Then for any $t>0$, the solution $y_{r}$ to the Equation (4.2) is nonexplosive up to any time $t_{0}<t$ for almost all $\hat{\omega}$ and $\omega$.

Proof. We fix $t>0$. Let

$$
\mathcal{E}_{t}=\mathrm{e}^{-\frac{1}{2} \int_{0}^{t} k^{2}(r) d r+\int_{0}^{t} k(r) d W_{r}}
$$

and

$$
\mathcal{V}(t, x)=u(t, x) \mathcal{E}_{t}^{-1}
$$

Then $\mathcal{V}$ satisfies the following reaction diffusion equation (see [25]),

$$
\begin{aligned}
\frac{\partial}{\partial t} \mathcal{V}(t, x) & =\frac{D}{2} \Delta \mathcal{V}+c\left(\mathcal{E}_{t} \mathcal{V}\right) \mathcal{V} \\
\mathcal{V}(0, x) & =u_{0}(x)=\chi_{x \leq 0}
\end{aligned}
$$


Applying Feynman-Kac formula, we have for any $x$,

$$
\begin{aligned}
\mathcal{V}(t, x) & =\hat{E} u_{0}\left(x_{t}\right) \mathrm{e}^{\int_{0}^{t} c\left(\mathcal{E}_{t-r} \mathcal{V}\left(t-r, x_{r}\right)\right) d r} \\
& \leq \mathrm{e}^{c(0) t} \hat{E} u_{0}\left(x_{t}\right) \\
& \leq M(t)
\end{aligned}
$$

Here $M(t)=\mathrm{e}^{c(0) t}, x_{t}=x+\sqrt{D} B(t), B(t)$ is a Brownian motion on the probability space $(\hat{\Omega}, \hat{F}, \hat{P})$ and $\hat{E}$ is the expectation with respect to $\hat{P}$. As $c(u)$ is decreasing, so

$$
c\left(\mathcal{E}_{t-r} \mathcal{V}\left(t-r, x_{r}\right)\right) \geq c\left(\mathcal{E}_{t-r} M(t-r)\right) .
$$

Therefore

$$
\begin{aligned}
\mathcal{V}(t, x) & \geq \hat{E} u_{0}\left(x_{t}\right) \mathrm{e}^{t} c\left(\mathcal{E}_{t-r} M(t-r)\right) d r \\
& =\mathrm{e}^{\int_{0}^{t} c\left(\mathcal{E}_{t-r} M(t-r)\right) d r} H(t, x)
\end{aligned}
$$

Here $H(t, x)$ is the solution of the heat equation $\frac{\partial}{\partial t} H(t, x)=\frac{D}{2} \Delta H(t, x)$ with $H(0, x)=\chi_{x \leq 0}$. It is easy to see that $H(t, x)$ is decreasing with respect to $x$, so for all $x \leq 0$,

$$
H(t, x) \geq H(t, 0)=\int_{-\infty}^{0} \frac{1}{\sqrt{2 \pi D t}} \mathrm{e}^{-\frac{y^{2}}{2 D t}} d y=\frac{1}{2} .
$$

It follows from (4.8) and (4.9) that for all $x \leq 0$,

$$
\mathcal{V}(t, x) \geq \frac{1}{2} \mathrm{e}_{0}^{t} c\left(\mathcal{E}_{t-r} M(t-r)\right) d r .
$$

On the other hand, $\mathcal{V}(t, x)$ satisfies

$$
\mathcal{V}(t, x)=\int_{R} p_{t}(y, x) u_{0}(y) d y+\int_{0}^{t} \int_{R} p_{t-r}(y, x) c\left(\mathcal{E}_{r} \mathcal{V}(r, y)\right) \mathcal{V}(r, y) d y d r
$$

where

$$
p_{t}(y, x)=\frac{1}{\sqrt{2 \pi D t}} \mathrm{e}^{-\frac{(y-x)^{2}}{2 D t}}
$$

Differentiating (4.11) with respect to $x$, we have

$$
\nabla_{x} \mathcal{V}(t, x)=\int_{R}\left(\nabla_{x} p_{t}(y, x)\right) u_{0}(y) d y+\int_{0}^{t} \int_{R}\left(\nabla_{x} p_{t-r}(y, x)\right) c\left(\mathcal{E}_{r} \mathcal{V}(r, y)\right) \mathcal{V}(r, y) d y d r
$$

It follows that there exists a constant $C>0$ such that

$$
|\nabla v(t, x)| \leq \int_{R} \frac{1}{\sqrt{D t}} \sqrt{\frac{(y-x)^{2}}{D t}} p_{t}(y, x) u_{0}(y) d y
$$




$$
\begin{aligned}
& +\int_{0}^{t} \int_{R} \frac{1}{\sqrt{D(t-r)}} \sqrt{\frac{(y-x)^{2}}{D(t-r)}} p_{t-r}(y, x) c\left(\mathcal{E}_{r} \mathcal{V}(r, y)\right) \mathcal{V}(r, y) d y d r \\
\leq & \frac{C}{\sqrt{t}} \int_{R} \frac{1}{\sqrt{4 \pi D t}} \mathrm{e}^{-\frac{(y-x)^{2}}{4 D t}} u_{0}(y) d y \\
& +\int_{0}^{t} \frac{C}{\sqrt{t-r}} \int_{R} \frac{1}{\sqrt{4 \pi D(t-r)}} \mathrm{e}^{-\frac{(y-x)^{2}}{4 D(t-r)}} d y \max \left\{c(0),\left|c\left(\mathcal{E}_{r} M(r)\right)\right|\right\} M(r) d r \\
\leq & \frac{C}{\sqrt{t}}+C \int_{0}^{t} \frac{\max \left\{c(0),\left|c\left(\mathcal{E}_{r} M(r)\right)\right|\right\} M(r)}{\sqrt{t-r}} d r .
\end{aligned}
$$

It then follows from (4.10) and (4.13) that for any $x \leq 0$,

$$
\begin{aligned}
|R(t, x)| & =\left|-\frac{\nabla u(t, x)}{u(t, x)}\right|=\left|-\frac{\nabla \mathcal{V}(t, x)}{\mathcal{V}(t, x)}\right| \\
& \leq \frac{\frac{C}{\sqrt{t}}+C \int_{0}^{t} \frac{\max \left\{c(0),\left|c\left(\mathcal{E}_{r} M(r)\right)\right|\right\} M(r)}{\sqrt{t-r}} d r}{\frac{1}{2} \mathrm{e}_{0}^{t} c\left(\mathcal{E}_{t-r} M(t-r)\right) d r} \\
& \leq\left(\frac{2 C}{\sqrt{t}}+2 C E(t, \omega)\right) \mathrm{e}^{-\int_{0}^{t} c\left(\mathcal{E}_{t-r} M(t-r)\right) d r} .
\end{aligned}
$$

Here $E(t, \omega)$ is bounded for almost all $\omega$ and so $R(t, x)$ is bounded for almost all $\omega \in \Omega$ for any $x \leq 0$.

Now it is easy to see $\nabla \mathcal{V}(t, x) \leq 0$ (this is because $u(0, x)$ is nonincreasing and can be approximated by a regular and nonincreasing function. Then directly differentiating Equation (4.5) with the regular initial condition and using the Feynman-Kac formula, we can easily see the space derivative is nonpositive. The claim follows then from taking the limit.) Therefore $R\left(t-r, y_{r}\right) \geq 0$. Integrating (4.2), we have

$$
y_{s}=\sqrt{D} B_{s}-\int_{0}^{s} R\left(t-r, y_{r}\right) d r \leq \sqrt{D} B_{s} .
$$

That is to say $y_{s}$ cannot be explosive to $+\infty$ at any finite time $\hat{\omega}$ almost surely and for all $\omega$. But according to (4.14), the solution cannot be explosive to $-\infty$ at any time $s \leq t_{0}<t$ $\hat{\omega}$ almost surely and for almost all $\omega \in \Omega$ as the drift $-R\left(t-r, y_{r}\right)$ is bounded for almost all $\omega, y_{r}<0$ and $r \leq t_{0}<t$. Here $t_{0}$ is any time $t_{0}<t$.

Before we prove the main theorem of this section, we present some priori estimate for $v(t, x)$ which will be used in the proof. Here $v(t, x)$ was defined by eqauation (3.4). We don't strive to get the best possible estimates here. By comparison, under Conditions (i) and (ii), it is easy to see

$$
v(t, x) \leq 1
$$

Therefore $c(v) \geq 0$. It follows that

$$
v(t, x)=\hat{E} u_{0}\left(x_{t}\right) \mathrm{e}^{t} \int_{0}^{t} c\left(v\left(t-s, x_{s}\right)\right) d s-\frac{1}{2} \int_{0}^{t} k^{2}(s) d s
$$




$$
\begin{aligned}
& \geq \mathrm{e}^{-\frac{1}{2} \int_{0}^{t} k^{2}(s) d s} \hat{E} u_{0}\left(x_{t}\right) \\
& =\mathrm{e}^{-\frac{1}{2} \int_{0}^{t} k^{2}(s) d s} \int_{-\infty}^{0} \frac{1}{\sqrt{2 \pi D t}} \mathrm{e}^{-\frac{(y-x)^{2}}{2 D t}} d y \\
& =\mathrm{e}^{-\frac{1}{2} \int_{0}^{t} k^{2}(s) d s} \int_{-\infty}^{-\frac{x}{\sqrt{D t}}} \frac{1}{\sqrt{2 \pi}} \mathrm{e}^{-\frac{y^{2}}{2}} d y .
\end{aligned}
$$

It is easy to see if $x \leq 1$,

$$
\begin{aligned}
v(t, x) & \geq \frac{1}{\sqrt{2 \pi}} \mathrm{e}^{-\frac{1}{2} \int_{0}^{t} k^{2}(s) d s} \int_{-\infty}^{-\frac{1}{\sqrt{D t}}} \mathrm{e}^{-\frac{y^{2}}{2}} d y \\
& \geq \frac{1}{\sqrt{2 \pi}} \mathrm{e}^{-\frac{1}{2} \int_{0}^{t} k^{2}(s) d s} \frac{\sqrt{D t}}{D t+1} \mathrm{e}^{-\frac{1}{2 t}} .
\end{aligned}
$$

Here we used a wellknown inequality $\int_{a}^{\infty} \mathrm{e}^{-\frac{y^{2}}{2}} d y \geq \frac{1}{a+\frac{1}{a}} \mathrm{e}^{-\frac{a^{2}}{2}}$ (see e.g. [21]). Therefore if $x \leq 1$,

$$
\begin{aligned}
\log v(t, x) & \geq \log \frac{1}{\sqrt{2 \pi}}-\frac{1}{2} \int_{0}^{t} k^{2}(s) d s+\frac{1}{2} \log D t-\log (D t+1)-\frac{1}{2 D t} \\
& \geq \log \frac{1}{\sqrt{2 \pi}}-\log 2-\frac{1}{2} \log D t-\frac{1}{2} \int_{0}^{t} k^{2}(s) d s-\frac{1}{2 D t} .
\end{aligned}
$$

And if $x>1$,

$$
\begin{aligned}
v(t, x) & \geq \frac{1}{\sqrt{2 \pi}} \mathrm{e}^{-\frac{1}{2} \int_{0}^{t} k^{2}(s) d s} \frac{1}{\frac{x}{\sqrt{D t}}+\frac{1}{\frac{x}{\sqrt{D t}}}} \mathrm{e}^{-\frac{x^{2}}{2 D t}} \\
& =\frac{1}{\sqrt{2 \pi}} \frac{x \sqrt{D t}}{x^{2}+D t} \mathrm{e}^{-\frac{1}{2} \int_{0}^{t} k^{2}(s) d s-\frac{x^{2}}{2 D t}} .
\end{aligned}
$$

It turns out that for $1 \leq x \leq \gamma t$,

$$
\begin{aligned}
& \log v(t, x) \\
\geq & \log \frac{1}{\sqrt{2 \pi}}+\log x+\frac{1}{2} \log D t-\log \left(x^{2}+D t\right)-\frac{1}{2} \int_{0}^{t} k^{2}(s) d s-\frac{x^{2}}{2 D t} \\
\geq & \log \frac{1}{\sqrt{2 \pi}}-\log \left(\gamma^{2}+D\right)+\frac{1}{2} \log D-\frac{1}{2} \log t-\frac{1}{2} \int_{0}^{t} k^{2}(s) d s-\frac{\gamma^{2}}{2 D} t .
\end{aligned}
$$

We are going to prove the main theorem of this section. In addition to Conditions (i)-(iv), set

Condition (v). For any $u>0, c^{\prime}(u) \leq-d_{0}$ for a constant $d_{0}>0$.

Theorem 4.2 Assume Conditions (i)-(v). There exist $t_{0}>0$ and $C_{1}>0, C_{2}>0$ and $\delta>0$, such that,

$$
P\left\{\omega \in \Omega: \sup _{x \leq\left(\frac{b}{a} \gamma-h\right) t}\left|\frac{\nabla u(t, x)}{u(t, x)}\right| \leq C_{1} \exp \left\{-C_{2} \sqrt{t} \text { for all } t \geq T\right\}>1-\exp \{-\delta T\}\right.
$$


and

$$
\begin{aligned}
& P\left\{\omega \in \Omega: \int_{t}^{\infty} \sup _{x \leq\left(\frac{b}{a} \gamma-h\right) t}|\nabla u(s, x)| d s \leq C_{1} \exp \left\{-C_{2} \sqrt{t}\right\} \text { for all } t \geq T\right\} \\
> & 1-\exp \{-\delta T\}
\end{aligned}
$$

for any $T>t_{0}$, and $h>0$.

Proof. Recall $R=-D \nabla \log u, y_{s}$ is defined by (4.2) and $R$ satisfies (4.1). Similar to [28], applying Ito's formula, we can prove that $R\left(t-s, y_{s}\right) \exp \left\{\int_{0}^{s} c^{\prime}\left(u\left(t-r, y_{r}\right)\right) u\left(t-r, y_{r}\right) d r\right\}$ is a martingale with respect to $\hat{\mathcal{F}}_{s}$ on $\left(\hat{\Omega}, \hat{\mathcal{F}}, \hat{\mathcal{F}}_{s}, \hat{P}\right\}$ for $0 \leq s<t$. Then by martingale property we have for a.e. $\omega \in \Omega$,

$$
R(t, x)=\hat{E} R\left(\frac{t}{2}, y_{\frac{t}{2}}\right) \exp \left\{\int_{0}^{\frac{t}{2}} c^{\prime}\left(u\left(t-r, y_{r}\right)\right) u\left(t-r, y_{r}\right) d r\right\} .
$$

Note by integration by parts formula, Ito's formula and (4.1),

$$
\begin{aligned}
& \int_{0}^{\frac{t}{2}} R\left(t-s, y_{s}\right) \exp \left\{\int_{0}^{s} c^{\prime}\left(u\left(t-r, y_{r}\right)\right) u\left(t-r, y_{r}\right) d r\right\} d s \\
= & \frac{t}{2} R\left(\frac{t}{2}, y_{\frac{t}{2}}\right) \exp \left\{\int_{0}^{\frac{t}{2}} c^{\prime}\left(u\left(t-r, y_{r}\right)\right) u\left(t-r, y_{r}\right) d r\right\} \\
& -\int_{0}^{\frac{t}{2}}(s) \exp \left\{\int_{0}^{s} c^{\prime}\left(u\left(t-r, y_{r}\right)\right) u\left(t-r, y_{r}\right) d r\right\} \\
& \times\left[-\frac{\partial}{\partial s} R\left(t-s, r_{s}\right)+\sqrt{D} \nabla R\left(t-s, y_{s}\right) d B_{s}-\nabla R\left(t-s, y_{s}\right) R\left(r-s, y_{s}\right) d s\right. \\
& \left.+\frac{D}{2} \Delta R\left(t-s, y_{s}\right) d s+c^{\prime}\left(u\left(t-s, y_{s}\right)\right) u\left(t-s, y_{s}\right) d s\right] \\
= & \frac{t}{2} R\left(\frac{t}{2}, y_{\frac{t}{2}}\right) \exp \left\{\int_{0}^{\frac{t}{2}} c^{\prime}\left(u\left(t-r, y_{r}\right)\right) u\left(t-r, y_{r}\right) d r\right\} \\
& -\sqrt{D} \int_{0}^{\frac{t}{2}}(s) \exp \left\{\int_{0}^{s} c^{\prime}\left(u\left(t-r, y_{r}\right)\right) u\left(t-r, y_{r}\right) d r\right\} \nabla R\left(t-s, y_{s}\right) d B_{s} .
\end{aligned}
$$

It turns out that

$$
\begin{aligned}
R(t, x)= & \frac{2}{t} \hat{E} \int_{0}^{\frac{t}{2}} R\left(t-s, y_{s}\right) \exp \left\{\int_{0}^{s} c^{\prime}\left(u\left(t-r, y_{r}\right)\right) u\left(t-r, y_{r}\right) d r\right\} d s \\
& +\frac{2 \sqrt{D}}{t} \hat{E} \int_{0}^{\frac{t}{2}}(s) \nabla R\left(t-s, y_{s}\right) \exp \left\{\int_{0}^{s} c^{\prime}\left(u\left(t-r, y_{r}\right)\right) u\left(t-r, y_{r}\right) d r\right\} d B_{s} \\
= & \frac{2}{t} \hat{E} \int_{0}^{\frac{t}{2}} R\left(t-s, y_{s}\right) \exp \left\{\int_{0}^{s} c^{\prime}\left(u\left(t-r, y_{r}\right)\right) u\left(t-r, y_{r}\right) d r\right\} d s .
\end{aligned}
$$


Applying Cauchy-Schwarz inequality we have

$$
\begin{aligned}
|R(t, x)| \leq & \frac{\sqrt{2}}{\sqrt{t}}\left(\hat{E} \int_{0}^{\frac{t}{2}}<R\left(t-s, y_{s}\right), R\left(t-s, y_{s}\right)>\right. \\
& \left.\quad \exp \left\{2 \int_{0}^{s} c^{\prime}\left(u\left(t-r, y_{r}\right)\right) u\left(t-r, y_{r}\right) d r\right\} d s\right)^{\frac{1}{2}} .
\end{aligned}
$$

Now we establish some preliminary estimate for $R(t, x)$. Let $J(t, x)=-D \log u(t, x)$, then we can check that $J$ satisfies the following nonlinear stochastic Hamilton Jacobi Bellman equation,

$$
d J(t, x)+\frac{1}{2}|\nabla J(t, x)|^{2} d t=\frac{D}{2} \Delta J(t, x) d t+c(u) d t-\frac{1}{2} k^{2}(t) d t+k(t) d W_{t} .
$$

Applying Itô's formula to $J\left(t-r, y_{r}\right)$ for $0 \leq r<t$ we have

$$
\begin{aligned}
d J\left(t-r, y_{r}\right)=\quad & -\frac{\partial}{\partial r} J\left(t-r, y_{r}\right) d r+\nabla J\left(t-r, y_{r}\right)\left(\sqrt{D} d B_{r}-R\left(t-r, y_{r}\right) d r\right) \\
& +\frac{D}{2} \Delta J\left(t-r, y_{r}\right) d r \\
= & \sqrt{D} \nabla J\left(t-r, y_{r}\right) d B_{r}-\frac{1}{2}\left\langle\nabla J\left(t-r, y_{r}\right), \nabla J\left(t-r, y_{r}\right)\right\rangle d r \\
& -c\left(u\left(t-r, y_{r}\right)\right) d r+\frac{1}{2} k^{2}(t-r) d r-k(t-r) d W_{t-r} .
\end{aligned}
$$

For any integer $N$, let $\tau_{N}=\inf \left\{s \in[0, t]:\left|\nabla J\left(t-r, y_{r}\right)\right| \geq N\right\}$ and $\tau_{N}=\infty$ if $\left|\nabla J\left(t-r, y_{r}\right)\right|<$ $N$ for any $0 \leq s \leq t$. Therefore

$$
\begin{aligned}
& J\left(t-\left(\frac{t}{2} \wedge \tau_{N}\right), y_{\frac{t}{2} \wedge \tau_{N}}\right)-J(t, x) \\
= & \sqrt{D} \int_{0}^{\frac{t}{2} \wedge \tau_{N}} \nabla J\left(t-r, y_{r}\right) d B_{r}-\frac{1}{2} \int_{0}^{\frac{t}{2} \wedge \tau_{N}}\left\langle\nabla J\left(t-r, y_{r}\right), \nabla J\left(t-r, y_{r}\right)\right\rangle d r \\
& -\int_{0}^{\frac{t}{2} \wedge \tau_{N}} c\left(u\left(t-r, y_{r}\right)\right) d r+\frac{1}{2} \int_{0}^{\frac{t}{2} \wedge \tau_{N}} k^{2}(t-r) d r-\int_{0}^{\frac{t}{2} \wedge \tau_{N}} k(t-r) d W_{t-r} .
\end{aligned}
$$

Taking expectations and noticing that $\int_{0}^{\frac{t}{2} \wedge \tau_{N}} \nabla J\left(t-r, y_{r}\right) d B_{r}$ is a martingale we have

$$
\begin{aligned}
& \frac{1}{2} \hat{E} \int_{0}^{\frac{t}{2} \wedge \tau_{N}}\left\langle\nabla J\left(t-r, y_{r}\right), \nabla J\left(t-r, y_{r}\right)\right\rangle d r \\
= & J(t, x)-\hat{E} J\left(t-\left(\frac{t}{2} \wedge \tau_{N}\right), y_{\frac{t}{2} \wedge \tau_{N}}\right) \\
& -\hat{E} \int_{0}^{\frac{t}{2} \wedge \tau_{N}} c\left(u\left(t-r, y_{r}\right)\right) d r+\frac{1}{2} \int_{0}^{\frac{t}{2} \wedge \tau_{N}} k^{2}(t-r) d r-\int_{0}^{\frac{t}{2} \wedge \tau_{N}} k(t-r) d W_{t-r} .
\end{aligned}
$$


Let $N \rightarrow \infty$ we obtain

$$
\begin{aligned}
& \frac{1}{2} \hat{E} \int_{0}^{\frac{t}{2}}\left\langle\nabla J\left(t-r, y_{r}\right), \nabla J\left(t-r, y_{r}\right)\right\rangle d r \\
= & J(t, x)-\hat{E} J\left(\frac{t}{2}, y_{\frac{t}{2}}\right)-\hat{E} \int_{0}^{\frac{t}{2}} c\left(u\left(t-r, y_{r}\right)\right) d r \\
& +\frac{1}{2} \int_{0}^{\frac{t}{2}} k^{2}(t-r) d r-\int_{0}^{\frac{t}{2}} k(t-r) d W_{t-r} .
\end{aligned}
$$

From Condition (iii), it is easy to see $-c(u) \leq a(u-1)<a u$. Recall (4.17) and (4.18). It is easy to see that there exist constants $\delta>0, M>0$ and $\Omega_{T}^{1} \subset \Omega$ with $P\left(\Omega_{T}^{1}\right)>1-\exp \{-\delta T\}$ such that for $\omega \in \Omega_{T}^{1}$, and $t \geq T$, and $x \leq \gamma t$,

$$
\begin{aligned}
\inf _{s \leq s \leq t} \int_{0}^{s} k(r) d W_{r} & \geq-\frac{M}{8} t \\
-\log v(t, x) & \leq \frac{M}{8} t \\
\sup _{s \leq s \leq t} \int_{0}^{s} k(r) d W_{r} & \leq \frac{M}{4} t \\
-\hat{E} \int_{0}^{\frac{t}{2}} c\left(u\left(t-r, y_{r}\right)\right) d r & \leq a \hat{E} \int_{0}^{\frac{t}{2}} u\left(t-r, y_{r}\right) d r \\
& \leq a \int_{0}^{\frac{t}{2}} \sup _{y} u(t-r, y) d r \\
& \leq \frac{M}{4} t \\
\left|\int_{0}^{\frac{t}{2}} k(t-r) d W_{t-r}\right| & \leq \frac{M}{4} t .
\end{aligned}
$$

It follows from (4.24-4.26), (4.16) and (3.3) that if $\omega \in \Omega_{T}^{1}$, and $t \geq T$, and $x \leq \gamma t$,

$$
\begin{aligned}
J(t, x) & \leq \frac{M}{4} t, \\
-\hat{E} J\left(\frac{t}{2}, y_{\frac{t}{2}}\right) & \leq \frac{M}{4} t .
\end{aligned}
$$

It turns out from (4.23), (4.27-4.30) that if $\omega \in \Omega_{T}^{1}$, and $t \geq T$, and $x \leq \gamma t$, then

$$
\frac{1}{2} \hat{E} \int_{0}^{\frac{t}{2}}\left\langle\nabla J\left(t-r, y_{r}\right), \nabla J\left(t-r, y_{r}\right)\right\rangle d r \leq M t .
$$

Therefore by (4.21) we have if $\omega \in \Omega_{T}^{1}$, and $t \geq T$, and $x \leq \gamma t$,

$$
|R(t, x)| \leq 2 \sqrt{M}
$$

Now we are in a position to estimate $R(t, x)$ on the crest. Denote $y_{r}$ the solution of $(4.2)$ with $y_{0}=x=\left(\frac{b}{a} \gamma-h\right) t$. Denote $\mathcal{K}_{1}=\left\{(s, y): y \leq\left(\frac{b}{a} \gamma-\frac{1}{2} h\right) s\right\}$. Define for each $\omega \in \Omega_{T}^{1}$,

$$
\tau=\inf \left\{s:\left(t-r, y_{r}\right) \notin \mathcal{K}_{1}\right\} .
$$


Then if $\tau \leq \sqrt{t}$, the process $\left(t-r, y_{r}\right)$ meets the line $y=\left(\frac{b}{a} \gamma-\frac{1}{2} h\right) s$ with $y_{\tau}=\left(\frac{b}{a} \gamma-\right.$ $\left.\frac{1}{2} h\right)(t-\tau) \geq\left(\frac{b}{a} \gamma-\frac{1}{2} h\right)(t-\sqrt{t}) \geq\left(\frac{b}{a} \gamma-\frac{1}{4} h\right) t$ for $t>t_{0}$ where $t_{0}$ is a constant such that $\left(\frac{b}{a} \gamma-\frac{1}{2} h\right) \sqrt{t}<\frac{1}{4} h t$ for all $t>t_{0}$. Notice also (4.31), we have for $t>t_{0}$ (we require $t_{0}$ large enough such that $2 \sqrt{M t} \leq \frac{1}{8} h t$ for all $\left.T>t_{0}\right)$ and all $\omega \in \Omega_{T}^{1}$, and $t \geq T$,

$$
\begin{aligned}
& \hat{P}\{\tau \leq \sqrt{t}\} \\
\leq & \hat{P}\left\{\max _{0 \leq r \leq \sqrt{t}} y_{r} \geq \frac{1}{4} h t\right\} \\
= & \hat{P}\left\{\max _{0 \leq r \leq \sqrt{t}}\left\{\sqrt{D} B_{r}-\int_{0}^{r} R\left(t-s, y_{s}\right) d s\right\} \geq \frac{1}{4} h t\right\} \\
\leq & \hat{P}\left\{\max _{0 \leq r \leq \sqrt{t}} \sqrt{D} B_{r} \geq \frac{1}{8} h t\right\} \\
\leq & \exp \left\{-\frac{h^{2}}{128 D} t^{\frac{3}{2}}\right\} .
\end{aligned}
$$

Therefore we have for $T>t_{0}$ and each $\omega \in \Omega_{T}^{1}, t \geq T$,

$$
\begin{aligned}
R(t, x)= & \hat{E} R\left(t-\tau \wedge \frac{t}{2}, y_{\tau \wedge \frac{t}{2}}\right) \exp \left\{\int_{0}^{\tau \wedge \frac{t}{2}} c^{\prime}\left(u\left(t-r, y_{r}\right)\right) u\left(t-r, y_{r}\right) d r\right\} \\
= & \hat{E} \chi_{\tau \leq \sqrt{t}} R\left(t-\tau, y_{\tau}\right) \exp \left\{\int_{0}^{\tau} c^{\prime}\left(u\left(t-r, y_{r}\right)\right) u\left(t-r, y_{r}\right) d r\right\} \\
& +\hat{E} \chi_{\sqrt{t}<\tau \leq \frac{t}{2}} R\left(t-\tau, y_{\tau}\right) \exp \left\{\int_{0}^{\tau} c^{\prime}\left(u\left(t-r, y_{r}\right)\right) u\left(t-r, y_{r}\right) d r\right\} \\
& +\hat{E} \chi_{\tau>\frac{t}{2} t} R\left(\frac{t}{2}, y_{\frac{t}{2}}\right) \exp \left\{\int_{0}^{\frac{t}{2}} c^{\prime}\left(u\left(t-r, y_{r}\right)\right) u\left(t-r, y_{r}\right) d r\right\}
\end{aligned}
$$

It turns out using (4.31) that for $T>t_{0}$ and each $\omega \in \Omega_{T}^{1}, t \geq T$,

$$
\begin{aligned}
|R(t, x)| \leq & \sqrt{2 M} \hat{P}(\tau \leq \sqrt{t})) \\
& +\sqrt{2 M} \hat{E} \chi_{\sqrt{t}<\tau \leq \frac{t}{2}} \exp \left\{-d_{0} \sqrt{t}\left(\frac{1}{\sqrt{t}} \int_{0}^{\sqrt{t}} u\left(t-r, y_{r}\right) d r\right)\right\} \\
& +\sqrt{2 M} \hat{E} \chi_{\tau>\frac{t}{2} t} \exp \left\{-\frac{d_{0} t}{2}\left(\frac{1}{\frac{t}{2}} \int_{0}^{\frac{t}{2}} u\left(t-r, y_{r}\right) d r\right)\right\}
\end{aligned}
$$

Recall Theorem 3.4, then for $T>t_{0}$ and each $\omega \in \Omega_{T}^{1}, t \geq T$,

$$
\begin{aligned}
|R(t, x)| \leq & \sqrt{2 M} \exp \left\{-\frac{h^{2}}{128 D} t^{\frac{3}{2}}\right\} \\
& +\sqrt{2 M} \hat{E} \chi_{\sqrt{t}<\tau \leq \frac{t}{2}} \exp \left\{-d_{0} \sqrt{t}\left(\frac{1}{\sqrt{t}} \int_{0}^{\sqrt{t}} \inf _{y \leq\left(\frac{b}{a} \gamma-\frac{1}{2} h\right)(t-r)} u(t-r, y) d r\right)\right\} \\
& +\sqrt{2 M} \hat{E} \chi_{\tau>\frac{t}{2} t} \exp \left\{-d_{0} \frac{t}{2}\left(\frac{1}{\frac{t}{2}} \int_{0}^{\frac{t}{2}} \inf _{y \leq\left(\frac{b}{a} \gamma-\frac{1}{2} h\right)(t-r)} u(t-r, y) d r\right)\right\}
\end{aligned}
$$




$$
\begin{aligned}
\leq & \sqrt{2 M} \exp \left\{-\frac{\delta^{2}}{128} t^{\frac{3}{2}}\right\} \\
& +\sqrt{2 M} \exp \left\{-d_{0} \frac{1}{2 a}\left(c(0)-\frac{k^{2}}{2}\right) \sqrt{t}\right\} \\
& +\sqrt{2 M} \exp \left\{-d_{0} \frac{1}{2 a}\left(c(0)-\frac{k^{2}}{2}\right) \frac{t}{2}\right\} \\
\leq & C_{1} \exp \left\{-C_{2} \sqrt{t}\right\},
\end{aligned}
$$

for constants $C_{1}>0$ and $C_{2}>0$. That is to say for each $\omega \in \Omega_{T}^{1}$ and $T^{*}>t \geq T>0$,

$$
\begin{aligned}
& \int_{t}^{T^{*}} \sup _{x \leq\left(\frac{b}{a} \gamma-h\right) s}|\nabla u(s, x)| d s \\
\leq & \int_{t}^{T^{*}} C_{1} \exp \left\{-C_{2} \sqrt{s}\right\} \sup _{x \leq\left(\frac{b}{a} \gamma-h\right) s} u(s, x) d s \\
\leq & C_{1} \exp \left\{-\frac{1}{2} C_{2} \sqrt{t}\right\} \int_{t}^{T^{*}} \exp \left\{-\frac{1}{2} C_{2} \sqrt{s}\right\} d\left(\int_{t}^{s} \sup _{x \leq\left(\frac{b}{a} \gamma-h\right) r} u(r, x) d r\right) \\
\leq & C_{1} \exp \left\{-\frac{1}{2} C_{2} \sqrt{t}\right\} \exp \left\{-\frac{1}{2} C_{2} \sqrt{T^{*}}\right\} \int_{t}^{T} \sup _{x \leq\left(\frac{b}{a} \gamma-h\right) r} u(r, x) d r \\
& +\frac{1}{2} C_{1} C_{2} \exp \left\{-\frac{1}{2} C_{2} \sqrt{t}\right\} \int_{t}^{T^{*}} \frac{1}{\sqrt{s}} \exp \left\{-\frac{1}{2} C_{2} \sqrt{s}\right\} \int_{t}^{s} \sup _{x \leq\left(\frac{b}{a} \gamma-h\right) r} u(r, x) d r d s \\
\leq & C_{3} \exp \left\{-\frac{1}{2} C_{2} \sqrt{t}\right\} T^{*} \exp \left\{-\frac{1}{2} C_{2} \sqrt{T^{*}}\right\}+C_{3} \exp \left\{-C_{2} \sqrt{T^{*}}\right\},
\end{aligned}
$$

for a constant $C_{3}>0$. Therefore taking the limit $T^{*} \rightarrow+\infty$,

$$
\int_{t}^{\infty} \sup _{x \leq\left(\frac{b}{a} \gamma-h\right) s}|\nabla u(s, x)| d s \leq 2 C_{3} \exp \left\{-C_{2} \sqrt{t}\right\} .
$$

Remark 4.3 One can improve $\exp \left\{-C_{2} \sqrt{t}\right\}$ in (4.20) to $\exp \left\{-C_{2} t^{r}\right\}$ for any $0<r<1$.

Acknowledgement. The authors are grateful to D. Elworthy and S. Mohammed for helpful conversations. HZZ would like to acknowledge a grant from the Royal Society London which enabled him to visit University of Oslo where the paper was completed.

\section{References}

[1] L. Arnold (1998), Random Dynamical Systems, Springer-Verlag, Berlin.

[2] D. G. Aronson and H. F. Weinberger (1978), Multidimensional nonlinear diffusions arising in population genetics, Adv. in Math., Vol. 30, 33-76. 
[3] G. Barles, L. C. Evans and P. E. Sougandis (1990), Wave front propagation for reactiondiffusion system of PDE, Duke Math. J. Vol. 61, 835-38.

[4] H. Berestycki and L. Nirenberg (1992), Travelling fronts in cylinders, Ann. Inst. H. Poincaré, Anal. Non Linéaire, Vol. 9, 497-572.

[5] M. Bramson (1983), Convergence of solutions of the Kolmogorov equations to travelling waves, Mem. Amer. Math. Soc., Vol. 44, No. 285.

[6] A. Champneys and S. Harris and J. Toland and J. Warren and D. Williams (1995), Algebra, Analysis and Probability for a Coupled System of Reaction-Diffusion Equations, Phil. Trans. R. Soc. Lond. A, Vol. 350, 69-112.

[7] I.M. Davies, A. Truman and H.Z. Zhao (1996), Stochastic generalized KPP equations, Proc. R. Soc. Edinb. A, Vol. 126A, 957-983.

[8] K. D. Elworthy (1982), Stochastic Differential Equations on Manifolds, Cambridge University Press, Cambridge.

[9] K. D. Elworthy and A. Truman and H. Z. Zhao and J. G. Gaines (1994), Approximate travelling waves for generalized KPP equations and classical mechanics, Proc. $R$. Soc. Lond. A, Vol. 446, 529-554.

[10] K.D. Elworthy and H.Z. Zhao (1994), The propagation of travelling waves for stochastic generalized KPP equations, Mathematical and Cumputer Modelling, Vol. 20, No.4/5, 131-166.

[11] P. C. Fife (1979), Mathematical aspects of reacting and diffusing systems, Lecture Notes in Biomathematics, 28, Springer-Verlag, Berlin and New York.

[12] R. A. Fisher (1937), The Wave of Advance of Advantageous Genes. Ann. Eugenics, Vol. 7, 353-369.

[13] M. Freidlin (1985), Functional Integration and Partial Differential Equations, Princeton University Press, Precenton, New Jersey.

[14] M. Freidlin (1996), Markov Processes and Differential Equations: Asymptotic Problems, Birkhäuser, Basel.

[15] J. Gaines (1994), Appendix to [10].

[16] F. Hamel and N. Nadirashvili (1999), Entire solutions of the KPP equation, Comm. Pure Appl. Math., Vol. 52, 1255-1276.

[17] N. Ikeda and S. Watanabe (1981), Stochastic Differential Equations and Diffusion Processes, North-Holland Publishing Company. 
[18] A. Kolmogoroff, I. Petrovskii and N. Piscounoff (1937), Etude de l'equation de la Diffusion Avec Croissance de la Matiere et son Application a un Probleme Biologique. Moscow Uni., Bull. Math. 1, 1-25.

[19] X.M. Li and H.Z. Zhao (1996), Gradient estimates and the smooth convergence of approximate travelling waves for reaction-diffusion equations, Nonlinearity, Vol. 9, No.2, 459-77.

[20] P Malliavin and D. Stroock (1996), Short time behavior of the heat kernel and its logarithmic derivatives, J. Differential Geometry, Vol. 44, 550-70.

[21] H. P. McKean (1969), Stochastic Integrals, Academic Press, New York and London.

[22] H. P. McKean (1975), Application of Brownian motion to the equation of KolmogorovPetrovskii-Piskunov, Comm. Pure Appl. Math. Vol. 28, 323-331.

[23] S.-E. A. Mohammed and K.R. Scheutzow (2000), The stable manifold theorem for stochastic differential equations, Preprint.

[24] J. D. Murray (1993), Mathematical Biology, Springer-Verlag, Berlin.

[25] B. Øksendal, G. Våge and H.Z. Zhao (1999), Asymptotic properties of the solutions to stochastic KPP equations, Proc. R. Soc. Edinb., (to appear).

[26] S.-J. Sheu (1991), Some estimates of the transition density of a nondegenerate diffusion Markov process. Ann. Probab. 19 , no. 2, 538-561.

[27] J. Smoller (1983), Skock Waves and Reaction-Diffusion Equations, Springer-Verlag, New York.

[28] H.Z. Zhao (1997), On gradients of approximate travelling waves for generalized KPP equations, Proc. R. Soc. Edinb. A, Vol. 127A, No.2, 423-39.

B. Øksendal, Department of Mathematics, University of Oslo, Box 1053 Blindern, N-0316 Oslo, Norway and

Norwegian School of Economics and Business Administration, Helleveien 30, N-5035 BergenSandviken, Norway. email: oksendal@math.uio.no

G. Våge, Department of Mathematics, University of Oslo, Box 1053 Blindern, N-0316 Oslo, Norway.

H.Z. Zhao, Department of Mathematical Sciences, Loughborough University, Loughborough, Leicestershire, LE11 3TU, UK. email: H.Zhao@lboro.ac.uk 